\title{
The Ultraviolet Spectrograph on NASA's Juno Mission
}

\author{
G. Randall Gladstone · Steven C. Persyn · John S. Eterno • Brandon C. Walther • \\ David C. Slater • Michael W. Davis • Maarten H. Versteeg • Kristian B. Persson • \\ Michael K. Young • Gregory J. Dirks • Anthony O. Sawka • Jessica Tumlinson • \\ Henry Sykes · John Beshears • Cherie L. Rhoad • James P. Cravens • \\ Gregory S. Winters • Robert A. Klar • Walter Lockhart • Benjamin M. Piepgrass • \\ Thomas K. Greathouse • Bradley J. Trantham • Philip M. Wilcox • \\ Matthew W. Jackson • Oswald H.W. Siegmund · John V. Vallerga • Rick Raffanti • \\ Adrian Martin · J.-C. Gérard • Denis C. Grodent • Bertrand Bonfond • \\ Benoit Marquet • François Denis
}

Received: 16 August 2013 / Accepted: 17 February 2014 / Published online: 25 March 2014 (C) The Author(s) 2014. This article is published with open access at Springerlink.com

\begin{abstract}
The ultraviolet spectrograph instrument on the Juno mission (Juno-UVS) is a long-slit imaging spectrograph designed to observe and characterize Jupiter's far-ultraviolet (FUV) auroral emissions. These observations will be coordinated and correlated with those from Juno's other remote sensing instruments and used to place in situ measurements made by Juno's particles and fields instruments into a global context, relating the local data with events occurring in more distant regions of Jupiter's magnetosphere. Juno-UVS is based on a series of imaging FUV spectrographs currently in flight- the two Alice instruments on the Rosetta and New Horizons missions, and the Lyman Alpha Mapping Project on the Lunar Reconnaissance Orbiter mission. However, Juno-UVS has several important modifications, including (1) a scan mirror (for targeting specific auroral features), (2) extensive shielding (for mitigation of electronics and data quality degradation by energetic particles), and (3) a cross delay line microchannel plate detector (for both faster photon counting and
\end{abstract}

David C. Slater deceased 30 May 2011.

G.R. Gladstone $(\varangle) \cdot$ S.C. Persyn · J.S. Eterno · B.C. Walther · D.C. Slater · M.W. Davis ·

M.H. Versteeg · K.B. Persson · M.K. Young · G.J. Dirks · A.O. Sawka · J. Tumlinson · H. Sykes ·

J. Beshears · C.L. Rhoad · J.P. Cravens · G.S. Winters · R.A. Klar · W. Lockhart · B.M. Piepgrass ·

T.K. Greathouse · B.J. Trantham · P.M. Wilcox · M.W. Jackson

Southwest Research Institute, 6220 Culebra Road, San Antonio, TX, 78238, USA

e-mail: rgladstone@swri.edu

O.H.W. Siegmund $\cdot$ J.V. Vallerga $\cdot$ R. Raffanti $\cdot$ A. Martin

Sensor Sciences, 3333 Vincent Road, Pleasant Hill, CA, 94523, USA

J.-C. Gérard · D.C. Grodent · B. Bonfond

Laboratoire de Physique Atmosphérique et Planétaire, Université de Liège, Liège, Belgium

B. Marquet · F. Denis

Centre Spatial de Liège, Liège, Belgium 
improved spatial resolution). This paper describes the science objectives, design, and initial performance of the Juno-UVS.

Keywords Far-ultraviolet $\cdot$ Juno $\cdot$ Jupiter $\cdot$ Spectrograph

\section{Introduction}

Juno-UVS, the ultraviolet instrument on the Juno mission to Jupiter, is primarily based on the Alice instrument on the New Horizons (NH) mission to the Pluto system and on the Lyman Alpha Mapping Project (LAMP) instrument on the Lunar Reconnaissance Orbiter (LRO) mission currently in orbit around the Moon. Juno-UVS is an imaging spectrograph with a spectral range in the extreme-ultraviolet (EUV) and far-ultraviolet (FUV) of 68$210 \mathrm{~nm}$. This wavelength range was chosen to cover all important UV emissions from the $\mathrm{H}_{2}$ bands and the H Lyman series produced in Jupiter's auroras, while also including longer wavelengths sensitive to the absorption signatures of aurora-produced hydrocarbons. JunoUVS will remotely sense Jupiter's auroral morphology and brightness, providing context for in-situ measurements, and will map the mean energy and flux of precipitating auroral particles.

The Juno-UVS instrument was developed at SwRI and delivered to Lockheed Martin for integration onto the Juno spacecraft before launch on August 5, 2011. The instrument consists of two main assemblies: (1) a shoebox sized sensor, which includes a telescope section and a spectrograph \& detector section, and (2) an electronics box housed in the spacecraft vault. Besides this changed configuration (LRO-LAMP and the Alices each consisted of a single assembly), a number of changes have been incorporated to adapt the instrument to the Juno mission. A main design driver for these differences is Jupiter's harsh radiation environment. Another major change is the addition of a scan mirror, which allows the targeting of specific areas of interest when the spinning spacecraft is close to Jupiter. In the following sections we present the design and operation, the key changes from earlier designs, calibration results, and initial in-flight results for Juno-UVS, but we first begin with an overview of the science planned for Jupiter.

\section{Scientific Objectives}

Juno-UVS's main objective is to provide context for the particles and fields instruments (i.e., JADE, JEDI, Waves, and MAG) in the investigation of Jupiter's polar magnetosphere (Fig. 1). During the typically 6-hour near-perijove period of auroral observations, Juno-UVS will scan Jupiter's auroral regions once per 30-s spacecraft spin to observe the morphology, brightness, and spectral characteristics of Jupiter's far-ultraviolet (FUV) auroral emissions, which are primarily comprised of the Lyman series of $\mathrm{H}$ and the Lyman, Werner, and Rydberg band systems of $\mathrm{H}_{2}$. By obtaining time-tagged pixel list data (where each photon event is assigned a unique location, wavelength, and time), images and maps of the northern and southern auroral regions will be reconstructed on the ground at a resolution appropriate for the signal-to-noise ratio (SNR) of the spectral feature of interest. Near the beginning and end of the near-perijove observation period Juno-UVS will provide global snapshots of the northern and southern auroral morphology from a range of several Jovian radii. Closer to Jupiter, a scan mirror will be used to target the atmospheric region near the expected location of the Juno magnetic field line footprint (based on magnetic field models and the spacecraft 
Fig. 1 Artist conception of Juno emissions prominently displayed. While each $\sim 5000$-km altitude perijove occurs at relatively low latitudes, many auroral observations will be taken from within one Jovian radius ( $\left.R_{J}=71,492 \mathrm{~km}\right)$, allowing good spatial discrimination in orbit about Jupiter, with aurora

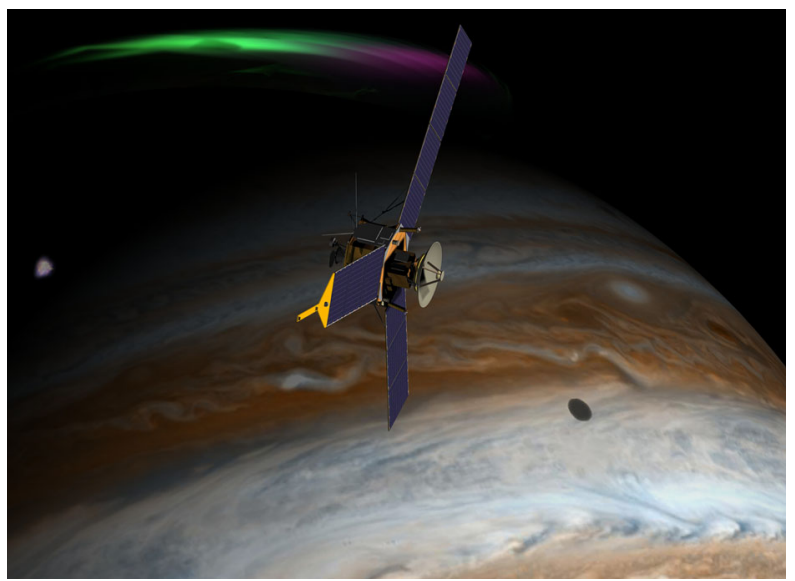

Fig. 2 Hubble Space Telescope image of Jupiter's northern FUV auroral emissions (Clarke et al. 2002). The three main auroral types are shown: (1) main oval, (2) polar emission, and (3) Galilean satellite magnetic field footprints. The brighter regions are several hundred kiloRayleighs (kR) or more

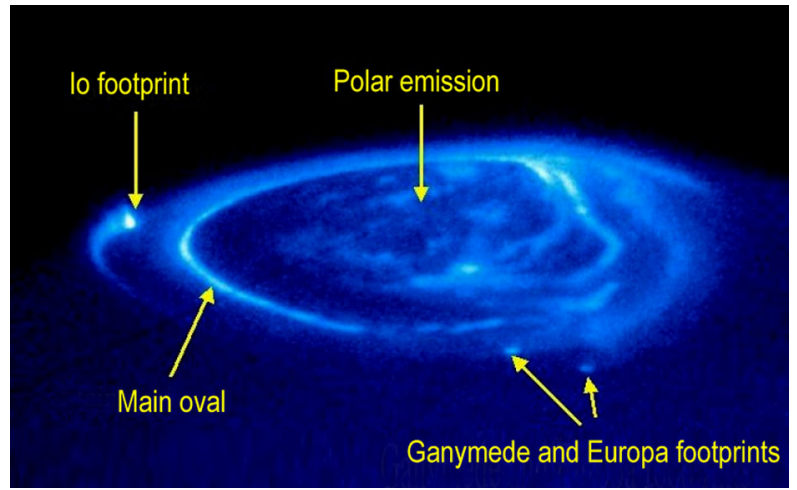

orbit and spin axis). This will allow a direct comparison of the precipitating particle fluxes measured by JADE and JEDI with the FUV emissions they produce upon impacting Jupiter's upper atmosphere, and how the particular region sampled by the spacecraft relates to the rest of the magnetosphere. Other frequent targets will be the magnetic field line footprints of the Galilean satellites (at a variety of local times and from near nadir to positions near the limb), the polar flares, and the main oval ansae (i.e., the locations where the main oval emission cross the limb). During MWR orbits (when the Juno spin and orbit planes coincide), the Juno-UVS scan mirror will be used much less, so that simultaneous FUV data will be acquired from the same auroral regions observed by the JIRAM near-IR instrument.

\subsection{Jupiter's FUV Auroral Emissions}

Jupiter's auroras have been usefully studied through FUV observations on several spacecraft (e.g., Voyager, Galileo, Cassini, New Horizons) and Earth-orbiting observatories (e.g., IUE, HST), and our understanding of them has improved dramatically, over the past few decades (e.g., Broadfoot et al. 1979; Ajello et al. 2001; Pryor et al. 2005; Gladstone et al. 2007; Prangé et al. 1996; Bhardwaj and Gladstone 2000; Clarke et al. 2004). Images from HST (see Fig. 2), in particular, have provided large datasets for studying auroral morphology and its spatial and temporal variability (e.g., Clarke et al. 1998, 2009; Nichols et al. 2007, 2009a, 2009b; Vogt et al. 2011). Likewise, FUV spectroscopy has provided strong constraints on the 
composition and temperature of Jupiter's upper atmosphere in the auroral regions and also on the energy and composition of the precipitating particles responsible for the aurora (e.g., Yung et al. 1982; Livengood et al. 1992; Trafton et al. 1998; Ajello et al. 2001, 2005; Gérard et al. 2003; Gustin et al. 2004, 2006). We now recognize four primary types or regions of Jovian aurora: (1) the main oval (or main emissions), driven by the breakdown of co-rotation in the plasma of the middle magnetosphere (e.g., Grodent et al. 2003a, 2004, 2008b; Radioti et al. 2008, 2009, 2010, 2011), (2) highly-variable polar emissions that are likely associated with reconnection regions near the dayside magnetopause or the flanks of the magnetotail (e.g., Pallier and Prangé 2001; Waite et al. 2001; Pryor et al. 2001, 2005; Gérard et al. 2003; Grodent et al. 2003b; Bonfond et al. 2011), (3) the emissions associated with the magnetic footprints of the Galilean satellites (e.g., Bonfond et al. 2009, 2012; Grodent et al. 2009; Wannawichian et al. 2010), and (4) the sub-auroral emissions equatorward of the main oval, including blobs attributed to plasma injections (e.g., Mauk et al. 2002; Bonfond et al. 2012) and diffuse emissions attributed to pitch angle diffusion of energetic electrons (e.g., Radioti et al. 2009).

Juno-UVS will provide new constraints on Jupiter's auroral emissions, not only regarding nightside morphology and spectroscopy (only observable by spacecraft), but also of crucial vertical structure-on certain orbits Juno-UVS will view the aurora at the limb from a distance of $\sim 30,000 \mathrm{~km}$, allowing a vertical resolution of $\sim 30 \mathrm{~km}$. In addition, high resolution Juno-UVS observations of the shape of the auroral oval with local time (with the help of magnetometer data) can address the need for a localized magnetic anomaly (e.g., Grodent et al. 2008a). In particular, Juno-UVS observations will provide color ratios for the components of the Jovian aurora for a variety of local times and viewing geometries (cf., Grodent et al. 1997; Gladstone et al. 2007). Examples of simulated Juno-UVS auroral-region targeting and further details on Juno auroral science are provided in Bagenal et al. (2014).

\subsection{Upper Atmosphere Structure and Composition}

Jupiter's upper atmosphere is currently poorly constrained, with the best available data from the Galileo probe which sampled the low-latitude regions (Seiff et al. 1998). Photochemical models have likewise concentrated on the low-latitudes (e.g., Gladstone et al. 1996; Moses et al. 2005), where the complexities of auroral region ion-neutral chemistry can be avoided (even though the auroral energy input globally dominates the actinic solar energy input, with $\sim 100$ TW continuously deposited into Jupiter's polar region atmosphere, e.g., Bhardwaj and Gladstone 2000). A few modeling studies of the chemistry and energetics in Jupiter's auroral regions exist (e.g., Perry et al. 1999; Wong et al. 2000, 2003; Melin et al. 2006), but more are needed.

Jupiter's upper atmosphere composition may be determined using Juno-UVS data in two different ways. In the auroral regions, the $\mathrm{H}_{2}$ Lyman band emissions typically originate somewhat below the homopause, and contain the absorption signature of overlying $\mathrm{CH}_{4}$ (giving rise to the color ratio) and even $\mathrm{C}_{2} \mathrm{H}_{2}$ and possibly other hydrocarbons (e.g., Dols et al. 2000). Also, in sunlit regions, the signal from reflected light at longer FUV wavelengths will show the absorption signatures of hydrocarbons, $\mathrm{NH}_{3}$, and possibly aerosols (e.g., Gladstone and Yung 1983). Finally, Juno-UVS observations each perijove (near sunset, at low-latitudes) are ideally suited to target airglow emissions, including ion recombination emissions similar to Earth's tropical arcs, which could constrain Jupiter's ionosphere structure and composition. Further details on Juno atmosphere science is provided in Ingersoll et al. (2014). Because of their intrinsic faintness (and the low duty cycle possible with the spinning Juno spacecraft), scientifically useful observations of Io Plasma Torus emissions or Galilean satellite emissions are unlikely. 

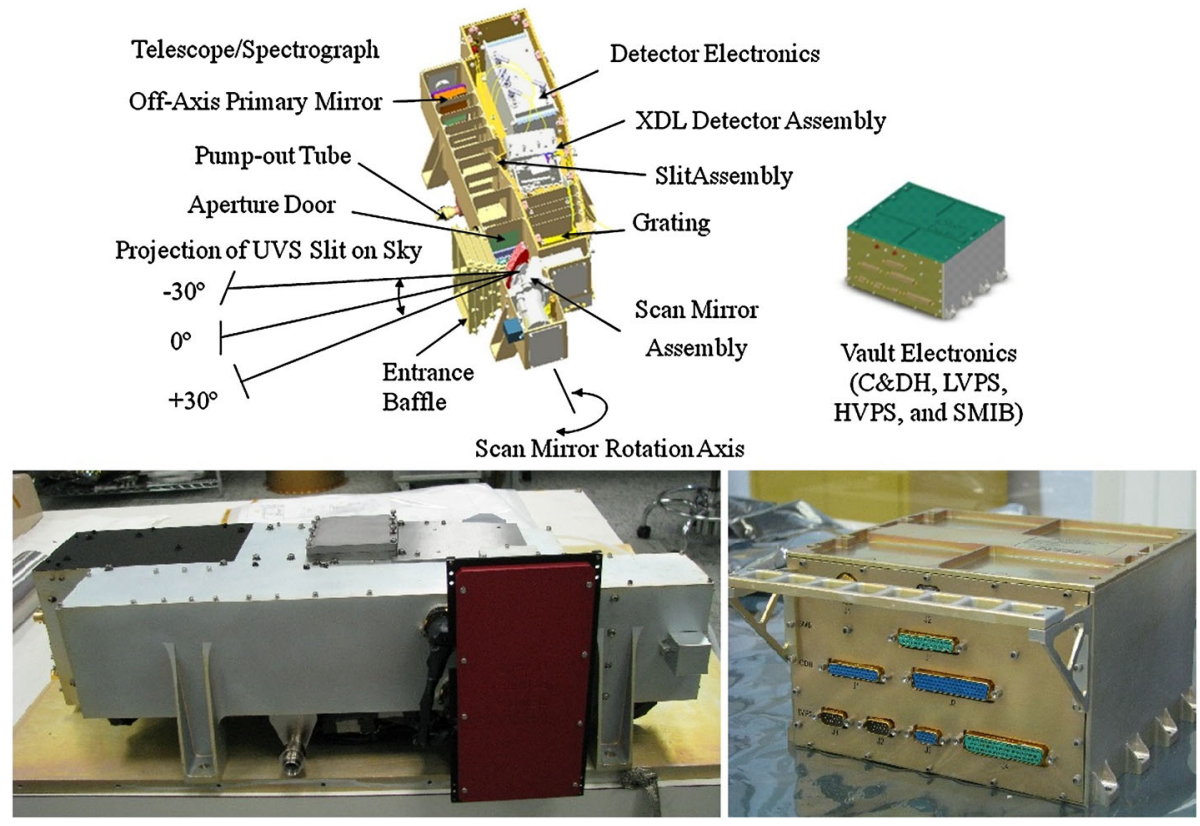

Fig. 3 Drawings of the Juno-UVS sensor (top left) and electronics box (top right), and pre-ship photographs of the sensor (bottom left) and electronics box (bottom right). Key sub-assemblies are indicated in the sensor drawing, and also show the operation of the scan mirror. The sensor photograph has the entrance baffle red tag cover in place for contamination control during spacecraft integration

\section{Instrument Description}

\subsection{Overview}

Juno-UVS is a photon-counting imaging spectrograph, based on the Lunar Reconnaissance Orbiter Lyman Alpha Mapping Project (LRO-LAMP), New Horizons-Alice (NH-Alice), and Rosetta Alice (R-Alice) instrument designs (Stern et al. 1998, 2008; Slater et al. 2001, 2005, 2008; Gladstone et al. 2010). Important changes from the earlier instruments include (1) a scan mirror (for targeting specific auroral features), (2) extensive shielding (for mitigation of electronics and data quality degradation by energetic particles), and (3) a cross delay line (XDL) microchannel plate (MCP) detector (for both faster photon counting and improved spatial resolution). In this section we provide a description of the instrument, highlighting these differences with previous designs.

Juno-UVS consists of two separate sections: a dedicated telescope/spectrograph assembly and a vault electronics box (see Fig. 3). The telescope/spectrograph assembly contains a telescope that feeds a $15 \mathrm{~cm}$ Rowland circle spectrograph with a spectral bandpass of 68$210 \mathrm{~nm}$. The telescope has an input aperture $4 \times 4 \mathrm{~cm}^{2}$ and uses an off-axis parabolic (OAP) primary mirror. A flat scan mirror situated at the front end of the telescope (used to look at up to $\pm 30^{\circ}$ perpendicular to the Juno spin plane) directs incoming light to the primary. The light is then focused onto the spectrograph entrance slit, which has three contiguous segments with fields-of-view (FOV) of $0.2^{\circ} \times 2.5^{\circ}, 0.025^{\circ} \times 2^{\circ}$, and $0.2^{\circ} \times 2.5^{\circ}$ projected onto the sky. This "dog bone" shaped slit's long axis is parallel to the spacecraft spin axis. Light 
entering the slit is dispersed by a toroidal diffraction grating which focuses the UV bandpass onto a curved microchannel plate (MCP) cross delay line (XDL) detector with a solar blind, UV-sensitive CsI photocathode. Tantalum plates contiguously surround the detector and detector electronics assembly to shield the detector from general particle radiation and high-energy electrons in particular. The detector electronics are located behind the detector.

The vault electronics box (Ebox) is located in the Juno spacecraft vault. Inside are two redundant high-voltage power supplies (HVPS), two redundant low-voltage power supplies (LVPS), the command and data handling (C\&DH) electronics, heater/actuator activation electronics, scan mirror electronics, and event processing electronics.

The overall function of the instrument is under the control of the C\&DH electronics, which consists of an 8-bit 8051 equivalent micro-controller, 32 Kbytes of PROM, 128 Kbytes of EEPROM, 128 Kbytes of SRAM, EEPROM and SRAM EDAC, and system timing functions. The $\mathrm{C} \& \mathrm{DH}$ accepts commands from the spacecraft and provides engineering telemetry while performing the following functions: (a) controlling the gain of the detector by adjusting the voltage that the HVPS provides to the MCP stack in the detector; (b) monitoring the detector MCP strip current, HVPS output voltage, and detector count rate so that it can "safe" the instrument upon an anomalous condition; (c) controlling the background radiation sensitivity of the XDL detector by adjusting the upper and lower level discriminator inputs to ensure that signals below and above certain levels are not processed; (d) controlling the optics heaters and the instrument aperture door; (e) controlling the onetime actuators such as the detector vacuum door and the front aperture fail-safe door (if needed), the instrument door launch latch mechanisms; and (f) controlling the scan mirror mechanisms.

The detector electronics receive detected event pulses from the detector and provide a digital indication of the spatial location, spectral location, and pulse height of each event being processed to the $\mathrm{C} \& \mathrm{DH}$. Event processing electronics receive valid individual events and process them into a pixel list (a time ordered record of detected events). Special periodic data markers, called time hacks, are inserted in the pixel list to provide a fixed time reference for the events. Pixel lists are accumulated continuously in a "ping-pong" memory with the data in one memory bank being transmitted to the spacecraft over the science telemetry channel while the other memory is used to continue data acquisition and store detector events and time hacks. The last pixel list data are transmitted to the spacecraft upon a command to stop data acquisition.

\subsection{Opto-Mechanical Design Overview}

The scan mirror, OAP mirror, and diffraction grating are each constructed from monolithic pieces of aluminum, coated with electroless nickel and polished using low-scatter polishing techniques. The aluminum optics, in conjunction with the aluminum housing, form an athermal optical design. The scan mirror, OAP mirror, and diffraction grating are also each overcoated with sputtered $\mathrm{Al} / \mathrm{MgF}_{2}$ for optimum reflectivity within the Juno-UVS spectral bandpass. Besides using low-scatter optics, additional control of internal stray light is achieved using internal baffle vanes within both the telescope and spectrograph sections of the housing, a holographic diffraction grating with low scatter and near-zero line ghost problems, and an internal housing with alodyned aluminum surfaces (Jelinsky and Jelinsky 1987; Moldosanov et al. 1998). In addition, a zero order light trap has a black anodized Al coating with very low surface reflectance at EUV/FUV wavelengths. Figure 4 shows a labeled opto-mechanical schematic of the interior of the Juno-UVS instrument, with light rays illustrating the optical path. 


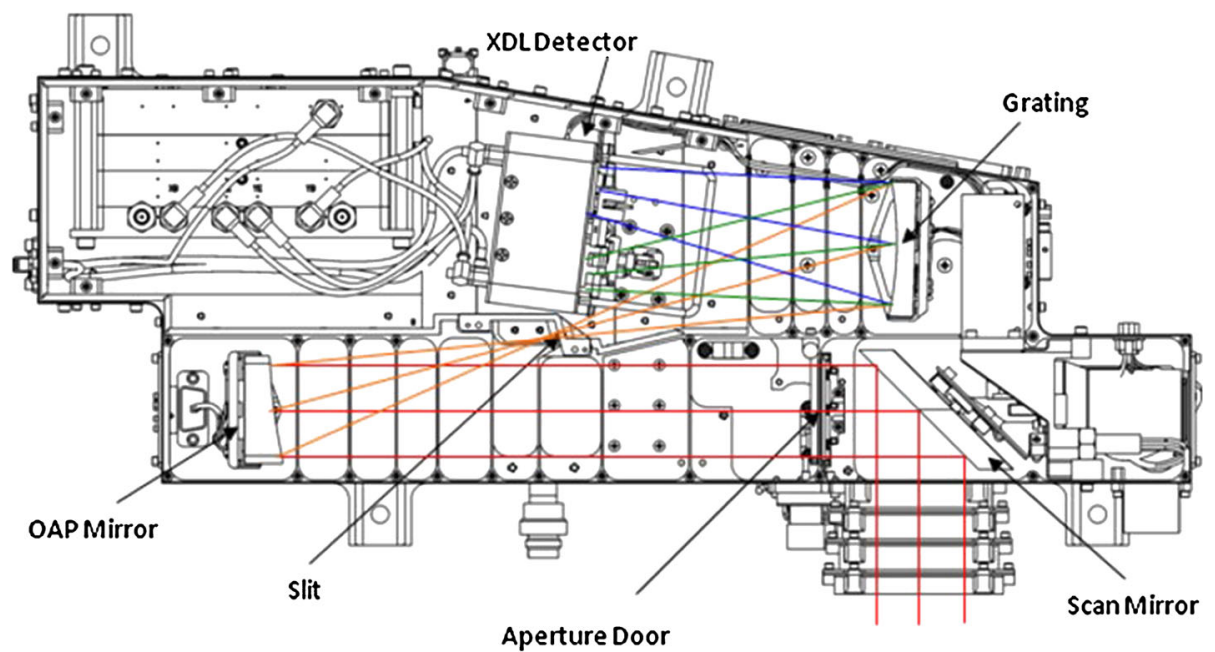

Fig. 4 An opto-mechanical schematic of the Juno-UVS sensor showing light rays traced through the main aperture door, reflecting from the OAP primary, passing through a focus at the slit, diffracted off the toroidal grating, and imaged onto the XDL MCP detector

\subsection{Detector and Detector Electronics}

The Juno-UVS detector configuration includes an XDL microchannel plate (MCP) detector scheme housed in a vacuum enclosure with a one-time opening door containing a UV-grade fused-silica window (for limited UV throughput during testing). The door was spring loaded for opening with a wax-pellet-type push actuator. The vacuum enclosure has a vacuum pump port and a small, highly polished region which functions as a zero-order reflector (directing zero-order light from the instrument grating into the zero-order trap on the side of the instrument housing). The vacuum enclosure also utilizes four female connectors for the anode signals, and two high-voltage (HV) connectors for the MCP and anode gap voltages.

The detector's MCP configuration uses a Z-stack that is cylindrically curved to match the 150-mm Rowland circle diameter to optimize spectral and spatial focus across the JunoUVS bandpass. The detector electronics provide two stimulation pixels that can be turned on to check data throughput and acquisition modes without the need to apply high voltage to the MCP stack or to have light on the detector. The MCP pulse-height information is output as 5 bits, which, together with the 11 bits of spectral and 8 bits of spatial information, results in the 3-byte output for every photon. The input surface of the Z-stack is coated with an opaque photocathode of CsI (Siegmund 2000).

A repeller grid above the curved MCP Z-stack enhances the detective quantum efficiency (DQE). Each of the three nested MCPs has a cylindrical 7.5-cm radius of curvature matching the instrument's Rowland circle radius (i.e., $15.0 \mathrm{~cm}$ diameter). The approximate resistance per MCP plate is $\sim 130 \mathrm{M} \Omega$. The MCP format is $4.6 \mathrm{~cm}$ wide in the spectral axis by $3.0 \mathrm{~cm}$ height in the spatial axis with $12-\mu \mathrm{m}$ diameter pores and a length-to-diameter (L/D) ratio of $80: 1$ per plate. The XDL anode is a rectangular format of $4.4 \mathrm{~cm} \times 3.0 \mathrm{~cm}$. The combination anode array and MCP sizes gives an active array format of $3.5 \mathrm{~cm} \times 1.8 \mathrm{~cm}$ necessary to capture the entire $68-210 \mathrm{~nm}$ instrument bandpass. The pixel readout format is 2048 pixels (spectral dimension) $\times 256$ pixels (spatial dimension). The active area is $3.5 \mathrm{~cm} \times 1.8 \mathrm{~cm}$, with $\sim 1500$ spectral pixels and $\sim 230$ spatial pixels. The XDL anode uses two orthogonal 
serpentine conductive strips for encoding an event's X-position and Y-position. Each event (i.e., a cloud of electrons exiting the MCP) is collected in equal amounts by the two strips, Charge is collected at each end of each strip, and the difference in arrival time at each end of a given strip is used to determine the event position (e.g., Siegmund et al. 1999).

The detector electronics are composed of a separate electronics package mounted directly behind the detector vacuum enclosure within the sensor housing. Power to the detector electronics is supplied by the Juno-UVS low voltage power supply (LVPS) and commandand-data handling electronics $(\mathrm{C} \& \mathrm{DH})$, both located in the electronics box (Ebox) in the spacecraft vault (several meters of cable away from the Juno-UVS sensor housing). The detector electronics are composed of five boards: (1) the amplifier board with two fast amps for the $X$ direction (spectral dimension) and two fast amps for the $Y$ direction (spatial dimension) and two charge amps for total event charge; $(2 \& 3)$ a time amplitude converter (TAC) board for each axis, $X$ and $Y$, that encodes 2048 pixels in the $X$-axis and 256 pixels in the $Y$-axis by event arrival time differences; (4) the digital board (DIG) that provides the control signals and interface logic, and (5) a delay line board to delay the End signals. The detector electronics also generate a 5-bit analog sum signal for each detected photon event that can be used for generating a pulse-height distribution (PHD) via ground test or flight analysis software. Pixel list data (i.e., a list of pixel $x, y$ addresses) is sent from the detector electronics to the $\mathrm{C} \& \mathrm{DH}$ electronics for further processing. A commandable stimulation pulse generator is also included that provides two stim pixels at two locations in the array; these are useful in checking data throughput without $\mathrm{HV}$ and in correcting for temperature effects on the wavelength scale.

A UV photon impinging on the photocathode generates a charge that is amplified by the microchannel plate Z-stack. The amplified charge cloud leaves the back end of the microchannel plate and is accelerated across the MCP-anode gap, impinging on the anode and generating pulses that propagate in both the $+X$ and $-X$ directions and $+Y$ and $-Y$ directions along separate integral delay lines to the detector electronics. The detector electronics then output the $X$ and $Y$ pixel locations to the C\&DH based on the time delay between the two opposing pulses in each axis.

The detector electronics require input DC voltages of $\pm 7.3 \mathrm{~V}$ and $+5.0 \mathrm{~V}$. The detector $\mathrm{MCP}$ high voltage is raised to a room temperature operational voltage of about $-4.2 \mathrm{kV}$. The gap between the MCP output and the anode array requires a voltage drop of approximately $-600 \mathrm{~V}$. Both the MCP and the anode gap voltages are supplied by the instrument's two redundant high voltage power supplies (HVPS) located in the Ebox. The overall detector gain is $\sim 2 \mathrm{E} 7( \pm 25 \%)$. At an expected average count rate of 2000 count/s, the amount of charge pulled from the MCP as a function of time is $\sim 0.2$ Coulomb/year.

\subsection{Instrument Electrical Design}

The Juno-UVS instrument support electronics are largely single-string, but include redundant features for certain important functions. These electronics include redundant lowvoltage power supplies (LVPS) and actuator electronics, the single-string command and data handling $(\mathrm{C} \& D H)$ electronics, the redundant optics decontamination heater system, and the redundant detector high-voltage power supplies (HVPS). All of these sub-systems are controlled by an Intel 8051 processor implemented in a radiation-hardened FPGA with $32 \mathrm{kB}$ of fuse programmable PROM, $128 \mathrm{kB}$ of EEPROM, $32 \mathrm{kB}$ of SRAM, and $128 \mathrm{kB}$ of acquisition memory. A detailed block diagram of these electronics is shown in Fig. 5. 


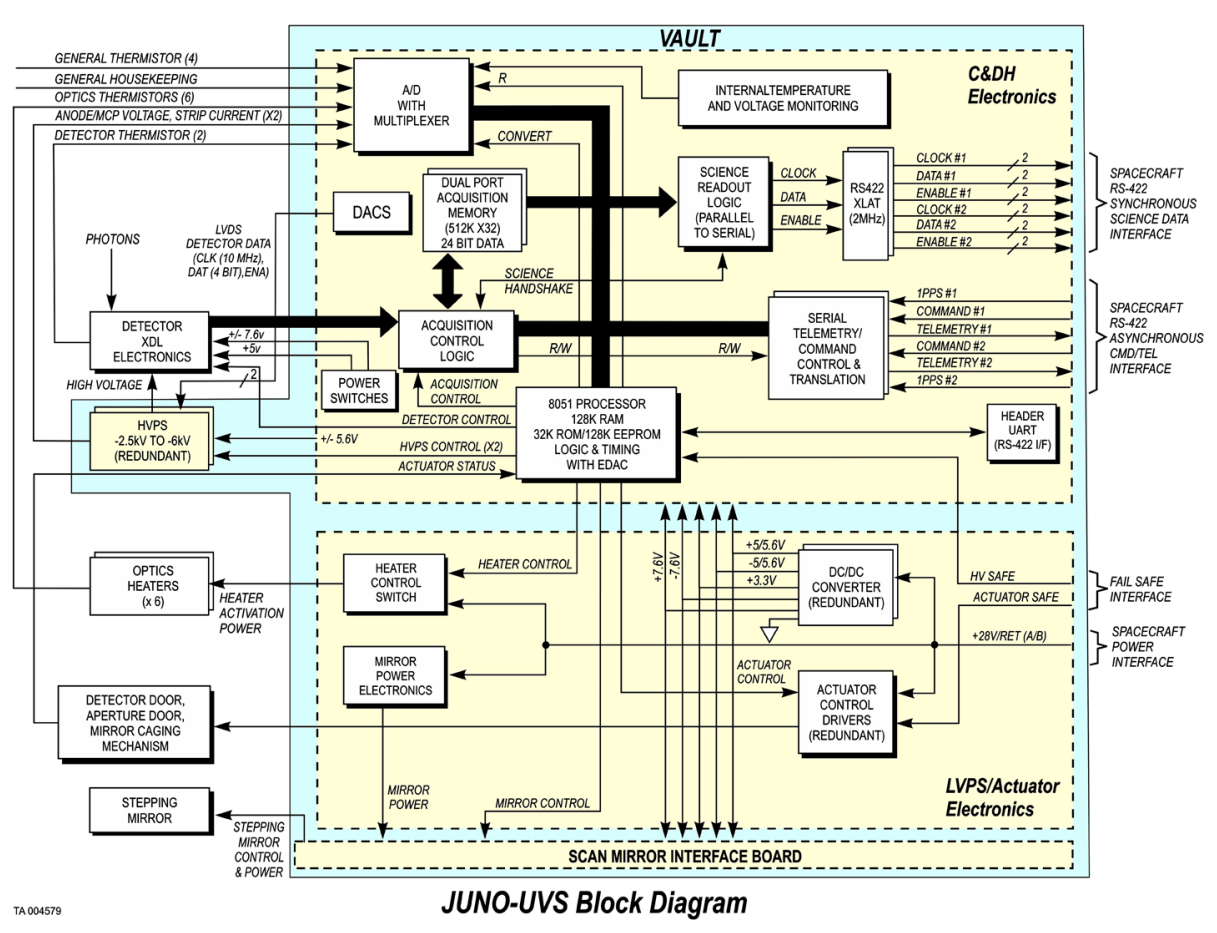

Fig. 5 Detailed block diagram of Juno-UVS

\subsection{Operations \& Data Collection Modes}

In the following subsections we present a detailed description of how Juno-UVS is operated, how science and housekeeping (HK) telemetry is produced, and how the instrument is commanded (including a separate account of how science data is acquired).

\subsubsection{Instrument Startup and Operational States}

Upon power-on or software reset, the software starts and performs a code check. Instrument power may be applied either to instrument power bus A or bus B. A code check determines which code will be started, either PROM or one of the four EEPROM pages. If no problems are detected in the code check of the PROM, execution always initially starts in PROM. If problems are detected, one of four EEPROM pages will be used as backup. After nominal startup, execution can also be directed to one of four EEPROM pages, to allow for flight software patches. After completion of this code check and clearing of the memory, the various software modules are initialized. This basic software initialization includes:

1. Enter safe state (deactivate any actuator operation)

2. Pre-set the parameter table with hard coded (nominal operational) values

3. Initialize parameter table (with majority-vote-based values from EEPROM)

4. Initialize sequence table (with majority-vote-based values from EEPROM)

5. Set the discriminator to its default value (based on parameter table)

6. Start the aperture closing operation (if enabled based on parameter file)

7. Determine the active command interface and initiate telemetry on the active side 


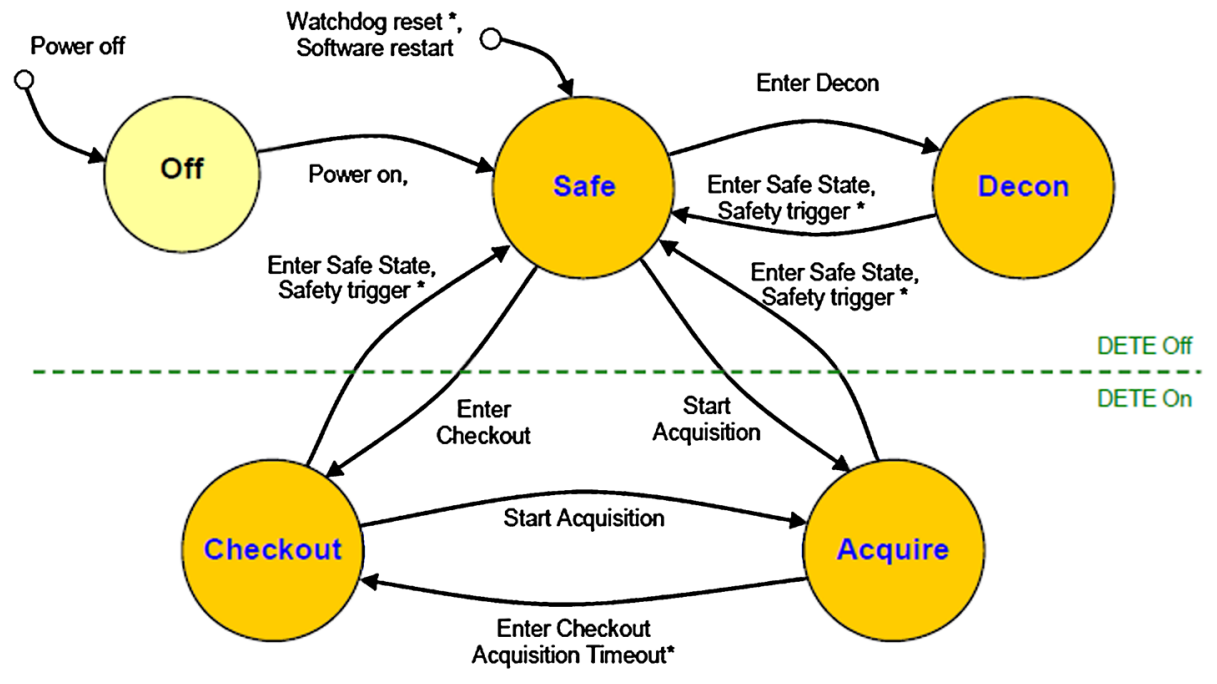

* Autonomous transitions: all other transitions are commanded

Fig. 6 Operational states diagram for Juno-UVS, showing the 5 instrument states and how they are connected. The 3 upper states (detector electronics off) are accessible with low-voltage alone, while the 2 lower states (detector electronics on) require high-voltage as well

After this initialization completes (in 3-4 seconds), the nominal operations of the instrument start and the software is ready to receive telecommands. The side selection is made either when a correct common interface protocol (CIP) message is received, or when 3 consecutive Time Sync Pulses (TSPs) are received at about the expected rate of $0.5 \mathrm{~Hz}$. Then telemetry for the active side is enabled and the instrument starts sending housekeeping packets. If after 180 seconds no side selection decision has been made, the instrument will start generating telemetry on the side specified by a configuration parameter. The selected side does not change until the instrument is power cycled.

Initially, the instrument clock begins counting from 1,000,000, incrementing every TSP by two counts. Whenever the first correct time synchronization message is received this counter is updated with the time value received from the spacecraft. If no TSPs are received, the instrument clock is updated based on the internal oscillator.

As indicated in Fig. 6, the Juno-UVS instrument can be in one of five states (including powered off) at any time. Four of these states are actually 'operational' states. Following power up or after a reset, the instrument will always enter the safe state. In the safe state, the instrument will always be idle; the HVPS, heaters and actuators will all be deactivated (except for a possible initial aperture door closing).

Changes between states can be either the result of received telecommands or performed autonomously by the instrument due to an unmasked safety trigger or a timed completion of an acquisition. The current state of the instrument is always reported in the housekeeping telemetry.

\subsubsection{Telemetry Generation}

Each operation cycle (nominally every 2 seconds), the Juno-UVS instrument generates a telemetry Internet Protocol/User Datagram Protocol (IP/UDP) formatted transaction. This 
transaction will at a minimum include a housekeeping (HK) packet and a 'safe-me' packet. Housekeeping packets are generated at a configurable periodic interval (nominally 5 cycles or 10 seconds). In between these housekeeping packets, the same telemetry format (and contents) will be generated; but the HK packet will be labeled as a no operation (NOOP) packet so that the spacecraft discards these packets (this allows Juno-UVS to pass a spacecraft aliveness test every 2 seconds, even at reduced HK rates). 'Safe Me' packets are normally deactivated by likewise disguising them as NOOP packets. Memory dump packets are generated whenever a memory dump has explicitly been requested by telecommand. Although after power-up the software will immediately start generating telemetry transactions, these transactions will initially not be sent to the spacecraft, as the telemetry buffers are not enabled. A buffer will nominally only be enabled once a side selection decision has been made, and will remain enabled until Juno-UVS is powered off.

Telemetry generation is a two-step process. After being triggered by the occurrence of a Time Sync Pulse (TSP) event, the Tm task starts gathering data from all the different sources within the system to fill the defined telemetry packet, and it is labeled as a HK or dummy NOOP packet, depending on the selected HK rate. Then a 'Safe Me' packet is added and nominally labeled as NOOP unless a safing condition exists. A memory dump packet may be generated, if requested, and if a generated memory-dump data set is available. The gathering of housekeeping data occurs at a high priority, so that HK data provides a detailed timecorrelated status of the instrument. After gathering all the data, the final IP/UDP protocol headers are generated by adding information such as length, the housekeeping packet, and checksums.

The actual transfer of the frame to the spacecraft is handed over to a lower priority task. This transfer can run at a lower priority as long as the transfer is completed well before the period expires. This transfer task moves the data to the Tm Universal Asynchronous Receiver-Transmitter (UART). The transmit UART includes a 32-byte FIFO buffer that is used to speed up Tm generation without too much processor activity. The processor activity is limited by waiting until FIFO almost empty status is reported in the Interrupt Service Routine (ISR). At that point, up to 20 bytes are copied into the UART buffer, without a need to perform separate checks on the intermediate buffer status (this allows for faster copying).

Included in the housekeeping telemetry is status reporting concerning received telecommands. Any command sent to the instrument will increment either the accepted or rejected command counter. This is also true for the 'Confirm Critical' command, even though this is only a partial command. An 'executed command counter' will be incremented only when a command completes execution without problems. These incrementing counters allow for the loss of a single Tm packet without a total loss of information. Also reported in the telemetry is the last detected error code. However, the Tm module simply reports the last received error code in the telemetry packet; so if multiple errors are detected in a given second, only the last (most recent one) will be reported. The most recent detected error will continue to be reported.

The configurable housekeeping telemetry rate allows for the adjustment of HK telemetry volume, based on the specific needs for an operational activity. The nominal housekeeping rate is one packet per 10 seconds. In this case, the packet contains all information that is needed for nominal operation use, including a vector of up to 101 samples of the instrument count rate determined at a $0.1 \mathrm{~s}$ rate. A higher rate may be selected when a faster response is desired; for instance, during ground testing or when troubleshooting problems. A lower rate may also be selected, for instance, when performing decontamination operations where only slowly-varying processes need to be monitored. This HK configurability can reduce the generated telemetry volume considerably (see Table 1). 
Table 1 Example HK generation rates

\begin{tabular}{|c|c|c|c|c|}
\hline Rate & $\begin{array}{l}\text { Max } \\
\text { (troubleshooting, } \\
\text { power up) }\end{array}$ & $\begin{array}{l}\text { Nominal } \\
\text { (nominal operations } \\
\& \text { science) }\end{array}$ & $\begin{array}{l}\text { Low } \\
\text { (decontamination) }\end{array}$ & $\begin{array}{l}\text { Very Low } \\
\text { (special) }\end{array}$ \\
\hline $\begin{array}{l}\text { HK sub-sample } \\
\text { rate }\end{array}$ & 1 & 5 & 120 & 240 \\
\hline TM rate & 1360 bits/s & 272 bits/s & 11.3 bytes/s & $5.7 \mathrm{bits} / \mathrm{s}$ \\
\hline HK volume & 612.0 kbytes/hour & 122.4 kbytes/hour & 5.1 kbytes/hour & 2.6 kbytes/hour \\
\hline $\begin{array}{l}\text { Relative TM } \\
\text { volume }\end{array}$ & $500 \%$ & $100 \%$ & $4 \%$ & $2 \%$ \\
\hline
\end{tabular}

\subsubsection{Telecommanding}

Juno-UVS operations are commanded using a set of 30 separate telecommands. Telecommand processing handles the redundant telecommand channels and includes error detection and recovery. Nominally, the spacecraft may send up to two telecommand transactions to the instrument every 2 s cycle. These are formatted as separate Internet Protocol/User Datagram Protocol (IP/UDP) Packets, and include (among other items) time and nadir messages.

The acceptance and completion status of the command execution is reported in the housekeeping data. The instrument verifies incoming telecommands before they can be executed; this basic verification includes a format and checksum check of the telecommand. As mentioned, Juno-UVS has two redundant telecommand interfaces, but after power-up, the active interface will be determined and operations from that point on will only use that single interface.

In addition to the command verification mechanism, the instrument implements two additional mechanisms to protect the instrument from anomalous telecommands. Some commands are only allowed when the instrument is in a specific state. In addition to this, a number of commands have been declared 'critical'. For Juno-UVS, this means that within a nominal 30-second timeout period, a specific confirmation command has to be received before the actual (critical) command execution starts. During most of the in-flight operations, this timeout is short compared with the light travel time to the spacecraft, meaning that the confirmation already has to be issued before confirmation of the acceptance of the command has been received on the ground. Still, this mechanism provides protection against accidental execution of commands.

The set of telecommands can be divided into three categories:

General operations - These allow for the complete basic operational commanding of the instrument. This includes setting and storing of parameters and starting and stopping of the science acquisitions. This set of seven commands allows for the full science operations of the instrument.

Manual operations-Additional capabilities needed during commissioning and instrument verification are provided by 15 additional telecommands that allow for extended command options. Some of these commands may be used for science operations depending on the situation.

Memory functions - Software code management and maintenance and additional debugging functions are provided by three general-purpose memory functions that allow for verification, load, and dump of memory blocks.

Whenever Juno-UVS detects errors while accepting or executing commands, an error will be reported in the generated telemetry packet. This includes an identifier for the telecom- 
mand (if available) and a general error code. The error code continues to be reported in the telemetry data until another error is detected or the instrument is reset. This simple form of error reporting is limited to reporting a single error per HK cycle (i.e., at most once per $2 \mathrm{~s}$ ). An additional mechanism implementing a small error log is available for more extensive problem investigation. The command code for any successful command is also reported in the telemetry data, so the telemetry registration can be used to reconstruct the received telecommands. Note that the parameters of a telecommand are not included in this reporting.

\subsubsection{Acquisition Commanding}

The basic science data acquisition function of Juno-UVS is handled by a start acquisition telecommand which has a number of parameters defining basic acquisition operations, with several settings defined by configuration parameters. At power-up the parameter list is initialized with values from non-volatile instrument memory. Once these general instrument configuration parameters have been set, these parameters are expected to need little change; and this mechanism results in simple acquisition command which only requires parameters that change frequently.

Once the acquisition start has been commanded, the instrument goes through an acquisition startup sequence, possibly including HV ramp up and aperture door commanding. The actual acquisition startup will start on the Juno synchronization signal (next TSP synchronization pulse), so the exact time of acquisition start is known and can be planned for.

Whether the aperture door is opened for a specific acquisition depends on the operational procedures. Periodically, the instrument will be commanded to acquire 'dark' acquisitions with the aperture door closed. For normal science acquisitions, the aperture door has to be open; this could either mean that the door is left open all the time or only commanded open for the specific acquisitions. At the end of an acquisition, the aperture door and the high voltage will be left at the latest commanded level.

For acquisitions, a science data frame will be generated whenever the acquisition memory is full of data (i.e., full of photon and/or time hack events). The time it takes for this memory to fill depends on the selected time hack rate, the brightness of the observed object (number of photons detected), the background event rate (primarily from penetrating energetic electrons at Jupiter), and the selected masking parameters. When an acquisition memory buffer is full, the acquisition will immediately continue in the next memory buffer while the filled buffer is transferred to the spacecraft. As this 'buffer full' event is related to the number of detected events, it is not synchronized to the instrument time sync. The transfer of a filled buffer to the spacecraft will be completed in about 0.4 seconds, so in nominal operations (if the photon and/or background count rates aren't too high) the buffer will be available for acquisition before the second buffer is filled. With the fixed buffer size and science data transfer rate, Juno-UVS can sustain a maximum transfer rate of 83,000 events/s to the spacecraft.

Nominally, the acquisition operation will continue until the instrument is explicitly commanded to 'stop acquisition.' In addition, a timeout mechanism is implemented that will terminate an acquisition mode when a predefined timeout period has expired; this mechanism may also be used to command acquisitions of a predetermined duration. When the acquisition is terminated, the data already in the acquisition buffer is sent to the spacecraft. Still a complete $32 \mathrm{k}$-event science frame is used even though it may only be partially filled. The used part in the pixel list frame can be recognized as the data consists of all 'zero' events 
which will normally not occur in the data (and will also be reported in the housekeeping packet). To command the 'stop acquisition' either the 'enter safe state' or 'enter checkout state' telecommand can be used. Both commands have the same effect, in that the acquisition is terminated. The 'enter safe state' will, in addition to terminating the acquisition, also deactivate the HVPS and close the aperture door.

For test purposes, an additional command parameter is available that allows for the selection of one out of five deterministic test patterns. The whole acquisition process proceeds just like a nominal acquisition, but initially the acquisition buffers are filled with the deterministic pattern. Since there are no actual data or time hacks involved, the acquisition buffer will not fill, so there are no hardware trigger moments in which the data is sent to the spacecraft. As a replacement, the software implements an exposure timer that controls the time at which the frame is actually sent (selectable from $0.1 \mathrm{~s}$ to $25 \mathrm{~s}$ ). The timeout period for this timer may even be varied during an acquisition to simulate a case where data generation rate varies over time.

\subsection{Changes from Previous LAMP and Alice Instruments}

Table 2 compares the key similarities and differences of Juno-UVS and its predecessors.

Table 2 R-Alice, NH-Alice, LAMP, and Juno-UVS Comparison

\begin{tabular}{|c|c|c|c|c|}
\hline Attribute & $\mathrm{R}$-Alice & $\mathrm{NH}$-Alice & LAMP & Juno-UVS \\
\hline Mass & $3.0 \mathrm{~kg}$ & $4.4 \mathrm{~kg}$ & $6.1 \mathrm{~kg}$ & $21.6 \mathrm{~kg}$ \\
\hline Power & $4.0 \mathrm{~W}$ & $4.4 \mathrm{~W}$ & $4.5 \mathrm{~W}$ & $9.8 \mathrm{~W}$ \\
\hline Dimensions & $\begin{array}{l}41 \times 20 \times \\
12 \mathrm{~cm}^{3}\end{array}$ & $46 \times 16 \times 12 \mathrm{~cm}^{3}$ & $55 \times 16 \times 12 \mathrm{~cm}^{3}$ & $\begin{array}{l}44 \times 24 \times \\
22 \mathrm{~cm}^{3}+13 \times \\
6 \times 9 \mathrm{~cm}^{3}(\text { Ebox })\end{array}$ \\
\hline Spectral Bandpass & $70-205 \mathrm{~nm}$ & $52-187 \mathrm{~nm}$ & $57-196 \mathrm{~nm}$ & $68-210 \mathrm{~nm}$ \\
\hline Input Channels & Airglow Only & $\begin{array}{l}\text { Airglow \& Solar } \\
\text { Occultation } \\
\text { Channel }\end{array}$ & $\begin{array}{l}\text { Airglow \& } \\
\text { Pinhole }\end{array}$ & Airglow Only \\
\hline Entrance Slit FOV & $\begin{array}{l}0.1^{\circ} \times 2.0^{\circ}+ \\
0.05^{\circ} \times 2.0^{\circ}+ \\
0.1^{\circ} \times 1.5^{\circ}\end{array}$ & $\begin{array}{l}0.1^{\circ} \times 4.0^{\circ}+ \\
2.0^{\circ} \times 2.0^{\circ}\end{array}$ & $0.3^{\circ} \times 6.0^{\circ}$ & $\begin{array}{l}0.2^{\circ} \times 2.5^{\circ}+ \\
0.025^{\circ} \times 2.0^{\circ}+ \\
0.2^{\circ} \times 2.5^{\circ}\end{array}$ \\
\hline $\begin{array}{l}\text { OAP Mirror \& } \\
\text { Grating Specs }\end{array}$ & \multicolumn{4}{|c|}{$\begin{array}{l}\text { Identical Specifications (except for optical coating) OAP: f/3, 120-mm focal length; } \\
\text { Grating: } 1600 \text { grooves } \mathrm{mm}^{-1}, 150-\mathrm{mm} \text { radius of curvature }\end{array}$} \\
\hline Optical Coating & $\mathrm{SiC}$ & $\mathrm{SiC}$ & $\mathrm{A} 1 / \mathrm{MgF}_{2}$ & $\mathrm{Al} / \mathrm{MgF}_{2}$ \\
\hline MCP Detector & DDL & DDL & DDL & XDL \\
\hline $\begin{array}{l}\text { Detector } \\
\text { Photocathodes }\end{array}$ & $\begin{array}{l}\mathrm{KBr} \\
(70-120 \mathrm{~nm}) \\
\mathrm{CsI} \\
(123-205 \mathrm{~nm})\end{array}$ & $\begin{array}{l}\mathrm{KBr} \\
(52-118.1 \mathrm{~nm}) \\
\mathrm{CsI} \\
(125.1-187 \mathrm{~nm})\end{array}$ & CsI (57-196 nm) & CsI $(68-210 \mathrm{~nm})$ \\
\hline $\begin{array}{l}\text { Ly } \alpha \text { Gap (i.e., bare } \\
\text { MCP) }\end{array}$ & $3 \mathrm{~nm}$ & $7 \mathrm{~nm}$ & No Gap & No Gap \\
\hline $\begin{array}{l}\text { Detector Door } \\
\text { Actuator }\end{array}$ & Dimple Motor & Wax Pellet & Wax Pellet & Wax Pellet \\
\hline $\begin{array}{l}\text { Pulse Height } \\
\text { Distribution Bins }\end{array}$ & 16 (per frame) & 64 (per frame) & 64 (per frame) & 17 (per event) \\
\hline HVPS & 1 & 2 (redundant) & 2 (redundant) & 2 (redundant) \\
\hline
\end{tabular}


Table 3 Vacuum radiometric test results summary

\begin{tabular}{|c|c|c|c|c|}
\hline Attribute & \multicolumn{2}{|c|}{ Required Performance } & \multicolumn{2}{|c|}{ Measured performance } \\
\hline $\begin{array}{l}\text { Dark Count } \\
\text { Rate }\end{array}$ & \multicolumn{2}{|c|}{$\leq 10$ counts/second (total array) } & \multicolumn{2}{|c|}{$<2$ counts/second (on ground) } \\
\hline Point Spread & \multicolumn{2}{|c|}{$<0.6 \mathrm{~nm}$ (spectral, point-source, on-axis) } & \multicolumn{2}{|c|}{$<0.6 \mathrm{~nm}$ (spectral, on-axis) } \\
\hline Function & \multicolumn{2}{|c|}{$<2.0 \mathrm{~nm}$ (spectral, filled slit) } & \multicolumn{2}{|c|}{$<1.1 \mathrm{~nm}$ (spectral, off-axis) } \\
\hline & \multicolumn{2}{|l|}{$<1^{\circ}$ (spatial) } & \multicolumn{2}{|c|}{$0.07^{\circ}-0.17^{\circ}$ (spatial) } \\
\hline $\begin{array}{l}\text { Wavelength } \\
\text { Bandpass }\end{array}$ & \multicolumn{2}{|c|}{ 78.0-172.0 nm (minimum coverage) } & \multicolumn{2}{|c|}{$67.5-210.0 \mathrm{~nm}$} \\
\hline $\begin{array}{l}\text { Spectral Plate } \\
\text { Scale }\end{array}$ & \multicolumn{2}{|c|}{$<0.095 \mathrm{~nm}$ per spectral pixel } & \multicolumn{2}{|c|}{$0.1020 \mathrm{~nm} \pm 0.0004 \mathrm{~nm}$} \\
\hline $\begin{array}{l}\text { Spatial Plate } \\
\text { Scale }\end{array}$ & \multicolumn{2}{|c|}{$<0.043^{\circ}$ per spatial pixel } & \multicolumn{2}{|c|}{$0.041^{\circ} \pm 0.001^{\circ}$} \\
\hline Off-Axis & \multicolumn{2}{|c|}{$P S T^{\mathrm{a}}<10^{-5}$ at $\theta_{\text {off }}>7^{\circ}$} & \multicolumn{2}{|c|}{$P S T<7 \times 10^{-6}$ at $\theta_{\text {off }}>7^{\circ}$} \\
\hline \multirow{7}{*}{$\begin{array}{l}\text { Absolute } \\
\text { Effective Area }\end{array}$} & Wavelength & Effective Area & Wavelength & Effective Area \\
\hline & $78-80 \mathrm{~nm}$ & $>0.002 \mathrm{~cm}^{2}$ & $92.6 \mathrm{~nm}$ & $0.0026 \pm 0.0003 \mathrm{~cm}^{2}$ \\
\hline & $100-110 \mathrm{~nm}$ & $>0.002 \mathrm{~cm}^{2}$ & $106.7 \mathrm{~nm}$ & $0.0042 \pm 0.0003 \mathrm{~cm}^{2}$ \\
\hline & $119-130 \mathrm{~nm}$ & $>0.2 \mathrm{~cm}^{2}$ & $121.6 \mathrm{~nm}$ & $0.54 \pm 0.08 \mathrm{~cm}^{2}$ \\
\hline & $130-140 \mathrm{~nm}$ & $>0.1 \mathrm{~cm}^{2}$ & $130.0 \mathrm{~nm}$ & $0.4 \pm 0.1 \mathrm{~cm}^{2}$ \\
\hline & \multirow[t]{2}{*}{$160-172 \mathrm{~nm}$} & $>0.02 \mathrm{~cm}^{2}$ & $140.0 \mathrm{~nm}$ & $0.18 \pm 0.09 \mathrm{~cm}^{2}$ \\
\hline & & & $168.5 \mathrm{~nm}$ & $0.017 \pm 0.004 \mathrm{~cm}^{2}$ \\
\hline
\end{tabular}

a $P S T=$ Point Source Transmittance, defined as irradiance at the focal plane divided by the input irradiance

\section{Calibration}

After it was assembled and through with environmental testing, just prior to delivery to Lockheed Martin for integration on the Juno spacecraft, Juno-UVS underwent a series of tests to characterize its radiometric performance. Specifically, the Juno-UVS flight instrument was tested in SwRI's vacuum radiometric calibration chamber in order to determine the best pre-launch values for: (1) dark count rate; (2) PSF and wavelength calibration; (3) off-axis light scatter, and (4) effective area. Note that some of these attributes (e.g., effective area) can be measured much more accurately in flight (through stellar calibration), while other important calibration data (e.g., flat field measurements) are obtained only after launch. Table 3 shows a summary of the results for each of the ground radiometric tests performed, along with the performance requirement. As shown in this table, all the specified radiometric requirements measured during the vacuum radiometric tests were met. Figure 7 shows Juno-UVS in the test chamber just before starting radiometric calibration (during 2010 October 12-17). The results of these ground calibrations are described in the following subsections.

\subsection{Dark Count Rate}

The Juno-UVS detector dark count rate was measured during ground calibration via a set of exposures that totaled 14.5 hours of accumulated exposure time. These datasets allowed us to examine both the temporal and spatial distribution of the dark noise and measure its rate. The time hack interval for the dark exposures was $0.512 \mathrm{~s}$. The average dark count 
Fig. 7 Juno-UVS just prior to undergoing testing in SwRI's vacuum radiometric calibration facility
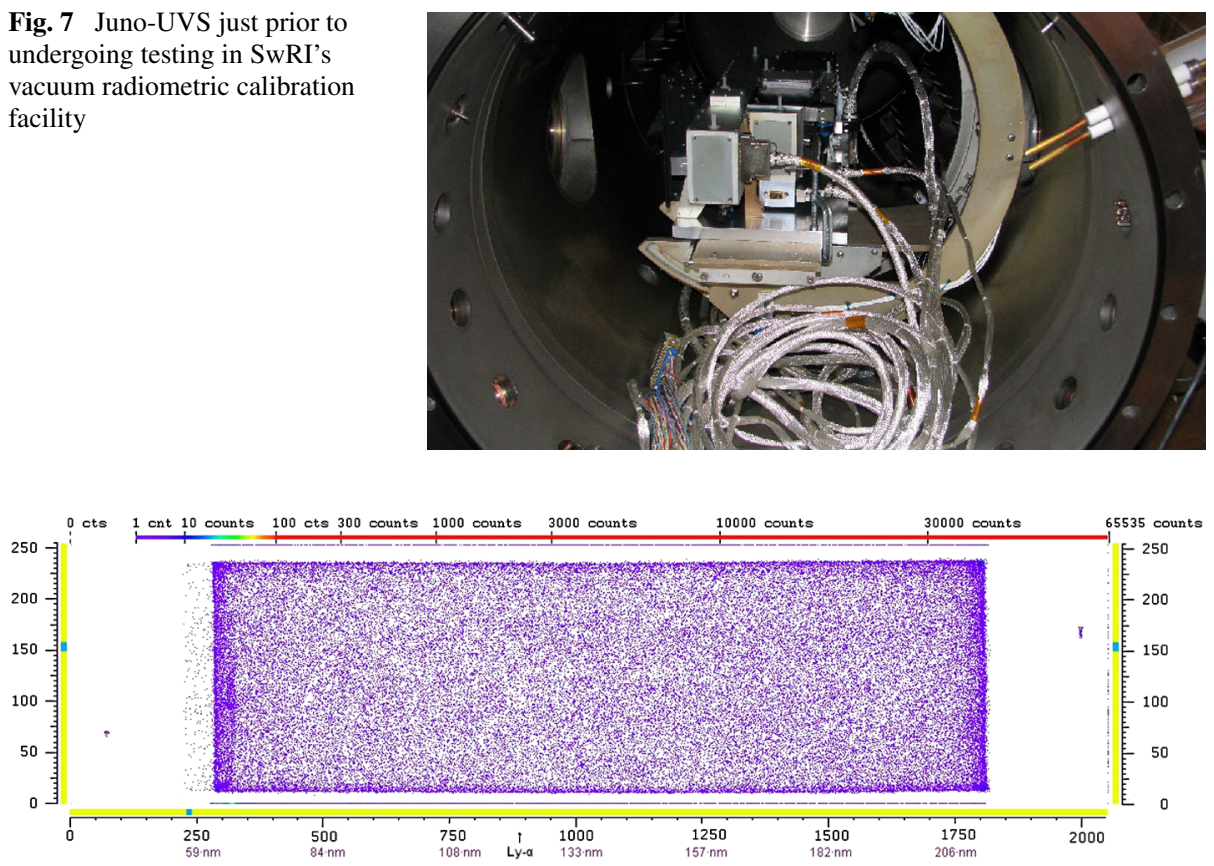

Fig. 8 Pre-launch background count test exposure, showing a dark rate of $\sim 2$ counts/s. This figure also shows how the raw Juno-UVS data appear when histogrammed in wavelength ( $x$ axis, $\sim 1500$ active pixels) versus slit position ( $y$ axis, $\sim 230$ active pixels). Event pileup occurs at the left and right edges of the detector, but the response over most of the active area is very uniform. The stim pulse locations are also seen, at the lower left and upper right

rate over the total 14.5-hour exposure interval varied between 1.5 and 2.0 counts/s (i.e., $0.18-0.26$ counts $\left.\mathrm{cm}^{-2} \mathrm{~s}^{-1}\right)$ with Poissonian counting statistics $(\sigma \sim 0.28$ counts/s averaged over 25 -s bins). This rate is well below the specified dark rate value of $<1$ count $\mathrm{cm}^{-2} \mathrm{~s}^{-1}$. Figure 8 shows a pre-launch spectral image of a dark exposure. Event pileup occurs at the left and right edges of the array, a characteristic of the XDL detector/electronics. This pileup results in a "warm" area in the lower right corner of the image that accumulates $5-10$ counts/pixel over 14.5 hours, or $\sim 2 \mathrm{E}-4$ counts/pixel/s. The two stim pixels are also visible at the lower left and upper right image edges.

\subsection{Spatial/Spectral Resolution}

The spectral and spatial PSFs were measured at $0.5^{\circ}$ intervals along the length of the slit and at specific wavelengths across the Juno-UVS bandpass using argon gas in a hollow-cathode UV light source. Point source pixel observations were acquired and converted via software into an image for each slit location beginning at the center of the slit (spatial offset of $0^{\circ}$ ) and at offset angles of $\pm 0.5^{\circ}, \pm 1.0^{\circ}, \pm 1.5^{\circ}, \pm 2.0^{\circ}, \pm 2.5^{\circ}$, and $\pm 3.0^{\circ}$ with respect to slit center. For each exposure, the image row with the maximum number of counts was used to fit a series of Gaussians for each identified emission line in the spectral axis. In the spatial axis each column was fit with a Gaussian to determine the spatial PSF. The measured values varied between 0.4 and $1.1 \mathrm{~nm}$, which satisfied the Juno-UVS specification of $<2.0 \mathrm{~nm}$ FWHM, and less than $0.6 \mathrm{~nm}$ for on-axis observations. 
The larger number and smaller size of the spatial pixels of the XDL detector versus the double-delay-line (DDL) detector used on previous instruments allows for proper sampling of the spatial PSF, as a point source is now imaged over multiple detector rows. The spatial resolution at the slit center well exceeds the specification of $1^{\circ}$ at all wavelengths. The best resolution is found near $90 \mathrm{~nm}$, which matches the theoretical astigmatic point of the JunoUVS optical design.

\subsection{Wavelength Calibration}

The images taken to determine the airglow PSF values (using the Ar lamp) were also used to determine the wavelength calibration at room temperature $\left(+22^{\circ} \mathrm{C}\right)$. Four principal argon emission lines at 91.9781, 93.2054, 104.822, and $106.666 \mathrm{~nm}$, two aluminum emission lines at 153.945 and $167.079 \mathrm{~nm}$, the $\operatorname{Ly} \alpha$ and $\operatorname{Ly} \beta$ hydrogen emissions at 121.567 and $102.572 \mathrm{~nm}$, the oxygen emission line at $130.22 \mathrm{~nm}$, the nitrogen emission line at $149.26 \mathrm{~nm}$, and the two bright neon emission lines at 73.5896 and $191.608 \mathrm{~nm}$ were used at slit center. The spectral plate scale and linear offset were determined with a simple linear fit to these data: $d \lambda / d x=0.1020 \pm 0.0004 \mathrm{~nm} /$ pixel; offset (slit center) $=31.73 \pm 0.43 \mathrm{~nm}$. The linear fit is good-the $\chi^{2}$ statistic for the fit is 6.15 with a linear correlation coefficient of 0.9998 . The standard deviation of the wavelength residual between the linear fit and the sixteen absolute emission line wavelengths is $\pm 0.636 \mathrm{~nm}$.

The total wavelength bandpass across the detector active area was computed with the above-measured plate scale and offset values. The active area starts at spectral pixel 300 and ends at pixel 1800. This area corresponds to a total wavelength bandpass of 67.5-210.0 nm, easily satisfying the minimal bandpass requirement of 78-172 nm.

The wavelength offset varies slightly with the temperature of the detector electronics at a measured rate of $\sim 0.6$ pixels $/{ }^{\circ} \mathrm{C}$ (i.e., wavelengths shift to the blue at a rate of $\sim 0.061 \mathrm{~nm} /{ }^{\circ} \mathrm{C}$; the plate scale variation with temperature is negligible and can be ignored). Care must thus be taken to apply the proper offset according to the detector electronics temperature for a given observation. This temperature dependence was measured/calibrated during detector subsystem and instrument thermal vacuum testing. The wavelength offset also varies with the $(x, y)$-location of the point source image within the slit due to both instrument pointing and to slit curvature aberrations. Additional wavelength calibration will be determined on orbit using standard UV stars.

\subsection{Scattered Light Characteristics}

The off-axis light scattering characteristics were measured in both the horizontal and vertical axes of Juno-UVS. Exposures using a collimated input source and a hollow-cathode lamp gas mixture of $\mathrm{H} / \mathrm{He}$ were acquired at angular input angles that varied between $-12^{\circ}$ and $+12^{\circ}$ with respect to the boresight axis in the horizontal plane (perpendicular to the slit length with the vertical axis fixed at the center of the slit), and the vertical plane (parallel to the slit length with the horizontal axis fixed at the center of the slit). The acquired images were analyzed to determine the point source transmittance $(P S T)$ as a function of incident input angle to the Juno-UVS boresight. The PST is defined as follows:

$$
P S T=\frac{E_{F P}}{E_{\text {input }}}
$$

where $E_{F P}$ is the irradiance at the focal plane; and $E_{\text {input }}$ is the input irradiance at the entrance aperture of the instrument. This expression can be written in terms of the measured off-axis 
angle count rate to the on-axis count rate ratio $\left(R_{\text {off }} / R_{0}\right)$; the effective area averaged over the Juno-UVS bandpass $\left(\left\langle A_{e f f}\right\rangle\right)$; the focal plane active area $\left(A_{F P}\right)$; the average in-band quantum efficiency of the CsI detector photocathode $(\langle Q E\rangle)$; and the ratio of the input beam area $\left(A_{\text {beam }}\right)$ to the geometric area $\left(A_{g}\right)$ of the entrance aperture (since the input beam underfills the entrance aperture). Using these parameters, the above equation for PST becomes

$$
P S T=\left(\frac{\left\langle A_{\text {eff }}\right\rangle}{A_{F P}\langle Q E\rangle}\right)\left(\frac{A_{\text {beam }}}{A_{g}}\right)\left(\frac{R_{\text {off }}}{R_{0}}\right) .
$$

The average effective area and quantum efficiency values used to compute the PST were measured during instrument radiometric and detector vendor tests $\left(\left\langle A_{\text {eff }}\right\rangle=0.54 \mathrm{~cm}^{2}\right.$; $\left.\langle Q E\rangle=0.25 ; A_{\text {beam }} / A_{g}=0.05 ; A_{F P}=7.5 \mathrm{~cm}^{2}\right)$. With these input values, we estimate a $P S T$ of 0.014 at an off-axis angle of $0^{\circ}$ (i.e., on-axis along the boresight with $R_{\text {off }} / R_{0}=1$ ). The requirement of $P S T<10^{-5}$ at off-axis angles $>7^{\circ}$ was met in both axes.

\subsection{Effective Area}

The effective area was measured across the Juno-UVS bandpass at discrete wavelengths using emission lines from neon, argon, and a hydrogen/helium gas mix covering a wavelength range of 73.6-191.6 nm. Exposures were made with the calibration chamber's monochromator set to the specific measurement wavelength. The monochromator output slit was set to provide a detector output count rate that did not show any saturation effects in the pulseheight distribution (since too high a local flux on the MCP stack causes a local gain drop evident in the pulse-height data). Background exposures were also taken for each illuminated exposure to allow for dark subtraction.

The beam flux was measured for each effective area measurement using a NISTcalibrated silicon ( $\mathrm{Si}$ ) photodiode. In most cases, the beam flux had to be increased in intensity to allow enough flux for a measurement with the photodiode. However, this increased level flux was too high for a measurement with Juno-UVS causing local MCP saturation. An Amptek channeltron was used to measure the ratio of the beam fluxes at both the lowand high-intensity levels to provide a correction factor to the flux measured by the NIST photodiode.

The equation used to compute $A_{\text {eff }}$ is

$$
A_{e f f}=\frac{A_{g} R(\lambda)}{\Phi(\lambda)},
$$

where $A_{g}$ is the geometric area of the Juno-UVS entrance aperture $\left(16 \mathrm{~cm}^{2}\right) ; R(\lambda)$ is the detector count rate (corrected for detector dead time and background); and $\Phi(\lambda)$ is the input flux to the instrument based on the NIST photodiode measurements.

Key to meeting the Juno-UVS sensitivity requirements is the choice of optical coatings for the grating, OAP mirror, and scan mirror. In the early mission planning stages, a SiC coating was planned, in order to provide good throughput at EUV wavelengths, where there are useful $\mathrm{H}_{2}$ emissions diagnostic of temperature and column density. Later, however, it was determined that the Juno-UVS wavelength resolution would not be high enough to make use of these features. Simulations indicated that $\mathrm{MgF}_{2}$-coated optics would result in a count rate $3 \times$ larger than $\mathrm{SiC}$-coated optics, for the same aurora. Thus, because of the low duty cycle available for observations with the spinning Juno spacecraft, the $\mathrm{MgF}_{2}$ optics were selected for flight, despite their lower sensitivity at wavelengths $<115 \mathrm{~nm}$. Figure 9 shows the current best estimate of the Juno-UVS effective area, as determined 
Fig. 9 Preliminary Juno-UVS effective area results from high-voltage checkout (HVCO) commissioning in December 2011 ( $\pm 1 \sigma$ region in $r e d)$, compared with model expectation (black), ground calibration with resonance lamps at specific wavelengths (blue), and the mission requirements (green). The HVCO results are based on International Ultraviolet Explorer (IUE) observations of several UV-bright stars which are used as flux standards

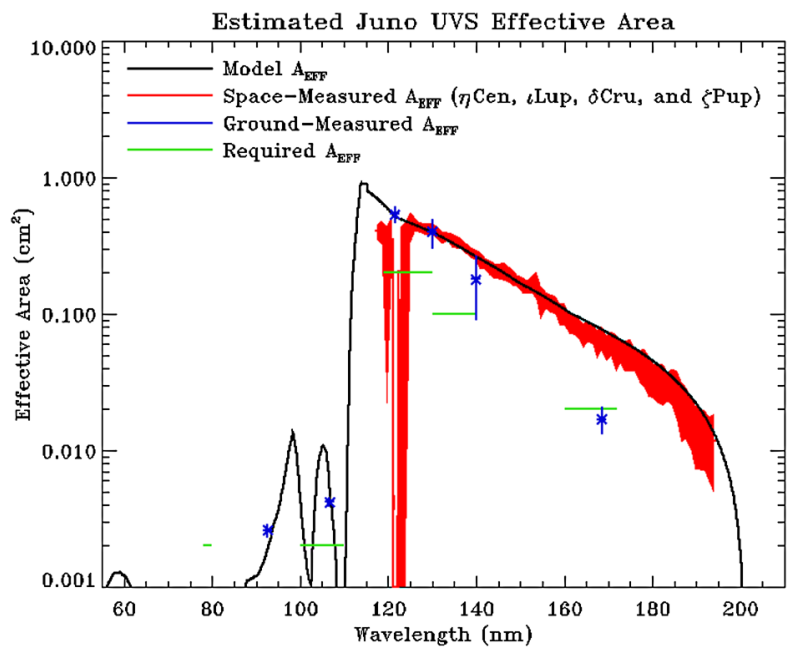

Estimated Juno-UVS Sensitivity

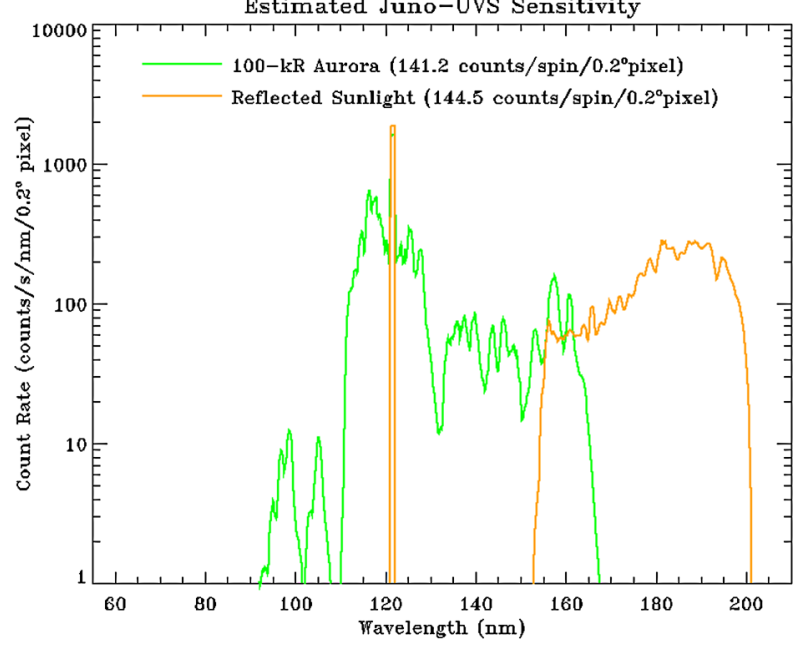

Fig. 10 Estimated response of Juno-UVS to (a) 100-kR Jupiter aurora of $\mathrm{H}_{2}$ and $\mathrm{H}$ emissions (green) and (b) reflected sunlight (orange) filling a solid angle of $1.2 \mathrm{E}-5$ steradians (appropriate for $0.2^{\circ} \times 0.2^{\circ}$ pixels). The model spectra are smoothed to the estimated filled-slit spectral resolution of $1.1 \mathrm{~nm}$. Integrated over the Juno-UVS bandpass, with an exposure time of $16.7 \mathrm{~ms}$ (appropriate for the time it takes Juno to spin $0.2^{\circ}$ at $2 \mathrm{RPM}$ ), a total count rate of 141.2 counts/spin $/ 0.2^{\circ}$ pixel is expected for a $100-\mathrm{kR}$ aurora, while 144.5 counts $/ \mathrm{spin} / 0.2^{\circ}$ pixel is expected for reflected sunlight, with the two sources largely separated by wavelength

by in-flight observations of selected UV-bright stars, and in comparison with requirements and ground measurements. Further in-flight observations, such as lunar observations during Earth flyby, will be used to improve the measured effective area at EUV wavelengths. As shown in Fig. 9 (and Table 3) the effective area requirements were met with significant margin between 110 and $165 \mathrm{~nm}$. Figure 10 shows the estimated response of Juno-UVS (using the in-flight effective area determination) to a modestly-bright 100-kR aurora (note that auroras of MR brightness are frequently observed with HST, e.g., Clarke et al. 2004) 


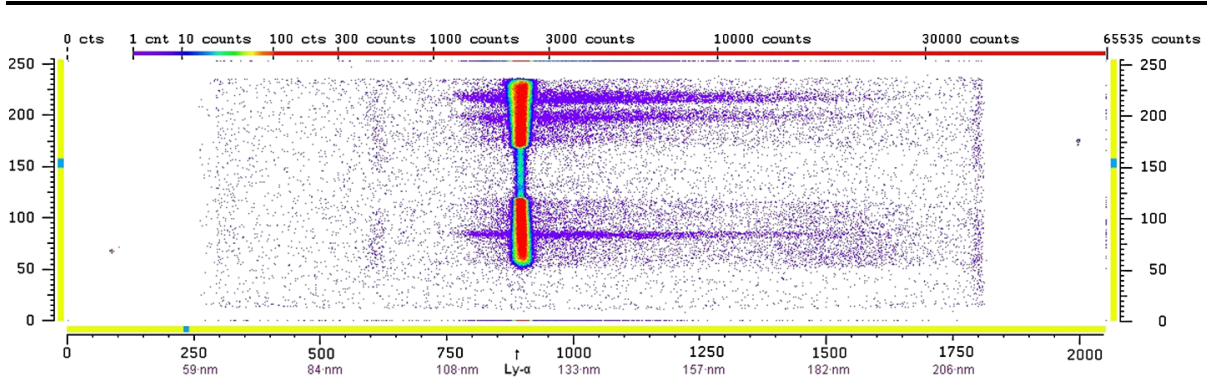

Fig. 11 "First-light" image, comprised of data from the first five spins of the spacecraft after Juno-UVS reached operational detector voltage. The slit with its "dog-bone" shape is clearly visible in the bright interplanetary Ly $\alpha$ spectral line at $121 \mathrm{~nm}$. Stars that briefly swept through the slit (due to the spin rate, a star is only in the narrow part of the slit for $8 \mathrm{~ms} / \mathrm{spin}$ ) have continuum spectra and appear as horizontal stripes

and to reflected sunlight. While there is overlap between the two sources, their contributions are expected to be easily separable.

\subsection{Commissioning}

During commissioning, yearly cruise calibrations, and at the apojoves of each Jupiter orbit, standard UV-bright stars will be observed to determine and monitor the effective area of Juno-UVS. These stellar sources will also be observed at various positions along the slit to provide accurate slit dimensions, vignetting information, and row-to-row variations in detector sensitivity. Filled-slit line shapes are acquired by looking at the interplanetary Ly $\alpha$ signal (e.g., Pryor et al. 2008). Juno-UVS "first-light" observations occurred during highvoltage checkout (HVCO), and are presented in Fig. 11. Also during high-voltage checkout, the optimal high voltage and discriminator settings are found, by inspection of a matrix of test observations and pulse height distributions (PHDs). The pulse height is obtained for every event, which makes monitoring PHDs much easier than in heritage versions of the Juno-UVS instrument.

Another important part of our in-flight calibration activities is to determine the digital dead time, which can decrease the measured signal at very large count rates. Ground tests by the detector provider indicate a digital dead time of $1.2 \mu$ s (i.e., corresponding to a $10 \%$ lower measured count rate for an input count rate $\sim 92,600$ counts/s; for comparison, the brightest UV star we will observe, $\beta$ Cru, is expected to have an input count rate of $\sim 1$ E6 counts/s). Initial results suggest that the ground dead time determination is accurate.

Finally, stellar observations are planned for use at Jupiter to measure exactly where JunoUVS is pointing, for any scan mirror position (the commanded scan mirror location is not determined on board by the instrument). By centroiding the stellar detections when the instrument is pointing away from Jupiter, we expect to locate photons to better than $0.05^{\circ}$ on the sky (the resolution of the narrow part of the slit). Figure 12 shows a "half-sky" map, in galactic coordinates, using data obtained during $\mathrm{HVCO}$ in which the scan mirror was stepped from $-30^{\circ}$ to $+30^{\circ}$ from the Juno spin plane.

\section{Data Products}

Juno-UVS data products, as delivered to NASA's Planetary Data System (PDS) for distribution to the public and archival purposes, include (1) Engineering Data Record (EDR) files 


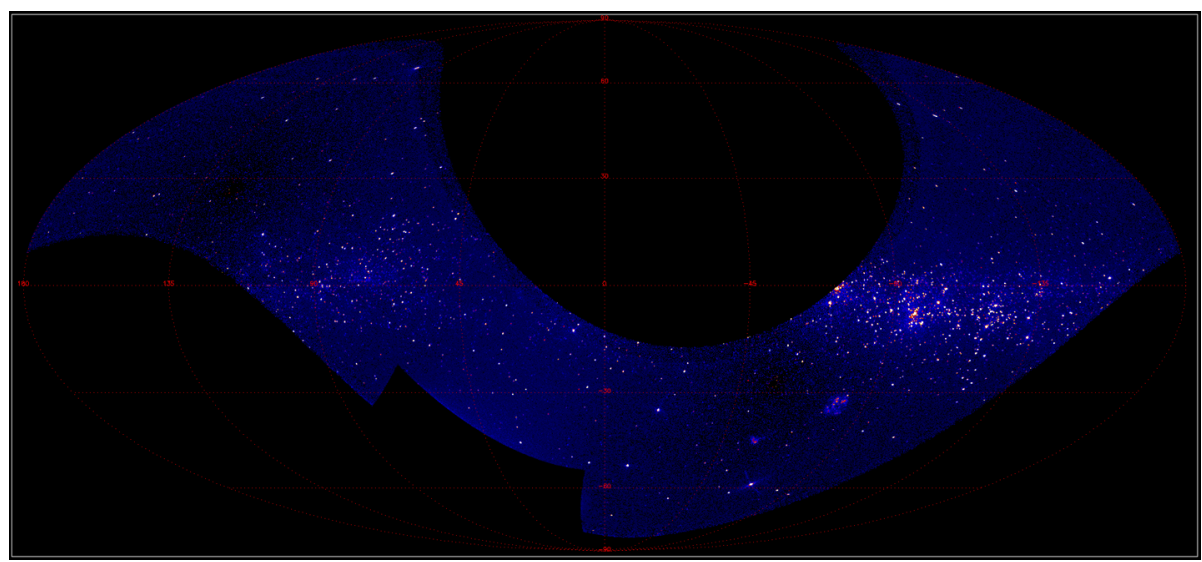

Fig. 12 Juno-UVS "half-sky" map showing the integrated starlight acquired during HVCO (for wavelengths $>125 \mathrm{~nm}$ ) in a Mollweide projection with galactic coordinates. The Large and Small Magellanic Clouds are evident in the lower right quadrant

(which contain minimally-processed Juno-UVS science and housekeeping (HK) telemetry files after they have been retrieved by the primary Juno-UVS SOC pipeline computer), (2) Reduced Data Record (RDR) files (which contain Juno-UVS data which have had the best available calibrations applied to bring them to physically useful units), and (3) high level data products (such as spectral images and maps) as they become available. A JunoUVS SOC pipeline "executive" program executes once per day to detect the newly delivered data files. After cataloging the received files, the "executive" program initiates execution of the Juno-UVS SOC data processing pipeline. The first element of the pipeline (nicknamed "Lima") is responsible for converting the raw data files into EDR data products (CODMAC Level 2). These products represent the lowest processing level of the Juno-UVS data and are delivered to the Planetary Data System (PDS) for archiving through the Juno Science Operations Center (JSOC). No calibrations are applied to the science data at this stage. Raw telemetry values are converted to engineering units where applicable; however, both raw and converted values are included in the EDR data product. Multiple versions of the output EDR products will be made available if software bugs affecting the output data are uncovered and corrected. In the event of an error whose correction alters released data, the data will be reprocessed by the revised software and then delivered to the PDS. The second element of the pipeline (nicknamed "Mike") calibrates and spatially locates the data contained in the EDR data products, resulting in the RDR data products (CODMAC Level 3). As with the EDR files, the RDR files are delivered to the Planetary Data System (PDS) for archiving through the JSOC.

\subsection{EDR Data Product Details}

The sources of the data contained in the Juno-UVS EDR data product are files containing (1) the Juno-UVS instrument low-speed housekeeping telemetry and (2) the Juno-UVS science data. These data are processed when both science and housekeeping files are present; if one is present without the other then a partial product will be created and that file will be flagged for later reprocessing. The data are grouped for specific observation events, and a formal description of the PDS-delivered data is provided in the associated EDR Software 
Interface Specification (SIS) document. The Juno-UVS EDR data product combines these files into a single FITS formatted file containing the following extensions:

Spectral vs. Spatial Image: This is a reconstructed histogram generated from the pixel list data in the science data file. Photon acquisition events are binned according to their spectral and spatial components. This summary image is used as a "quick-look" check on data quality. [Extension $0=$ primary FITS header and data unit (HDU)]

Spatial vs. Time Image: This is similar to the first dataset, but data are binned based on spatial and temporal parameters. The time dimension of the image covers $360^{\circ}$ on the sky, and is split into 5 panels of $72^{\circ}$ each. A new histogram is started each time the scan mirror position changes, as determined from the housekeeping data. This summary image is used as a "quick-look" check on data quality. [Extension 1]

Frame List: This dataset contains a list of the generated frame acquisitions. The list includes, for each frame, the instrument frame sequence number, start and stop times, tag bytes, quality factor and other instrument state information. The frame acquisition times and instrument state data contained in this list are used to cross-reference with the pixel list mode data for purposes of selecting data and checking timing consistency. [Extension 2]

Scan Mirror Data: This dataset contains a listing of all start and stop times when the scan mirror was at a fixed position. These data are taken from the housekeeping packets, so are limited to the HK rate selected for the given observations. [Extension 3]

Raw Frame Data: This dataset contains all the raw data from the science data file, except for the file header. [Extension 4]

Analog Count Rate: This dataset contains a sequence of time ordered photon count rates read from the housekeeping data packets. At nominal $\mathrm{HK}$ rates (down to $0.1 \mathrm{~Hz}$ ), this results in a 0.1-s sampling of the unmasked analog count rate. [Extension 5]

Digital Count Rate: Similar to the previous dataset, this contains the count rate as determined from the raw science data, as limited by masking and discriminator settings. These data are shown as counts/second. [Extension 6]

Pulse Height Distribution (Lyman Alpha): This is the first of three histograms where the bins are arranged as pulse height vs. time. One histogram will be created per spin. This histogram contains photons whose spectra are recorded on detector columns numbered between 850 and 930 (i.e., Ly $\alpha$ photons). [Extension 7]

Pulse Height Distribution (Stellar): This is the second of three histograms where the bins are arranged as pulse height vs. time. One histogram will be created per spin. This histogram contains photons whose spectra are recorded on detector columns numbered between 931 and 1770 (i.e., photons at wavelengths longer than Ly $\alpha$ ). [Extension 8]

Pulse Height Distribution (Stim): This is the last of three histograms where the bins are arranged as pulse height vs. time. One histogram will be created per spin. This histogram contains photons whose spectra are recorded on detector columns numbered between 0-149 and 1950-2047 (i.e., the stim pulse regions). [Extension 9]

Housekeeping Data: This dataset contains the complete housekeeping dataset, both in raw format and, where applicable, in calibrated engineering units. HK data are included here to assist with joint instrument and data quality trending analyses (foreseen and unforeseen). [Extension 10]

Parameter List: This table records the known values of the instrument parameter table, as reported in the housekeeping data. [Extension 11]

The primary data of the Juno-UVS EDR product files are the raw data frames in Extension 4 of the FITS files. These data frames contain a series of time-tagged UV photon detection events. The histogram image in the primary FITS header and data unit (HDU) are simply these photon events histogrammed into the 2048 spectral by 256 spatial bins. These 
histograms are included up front since most FITS viewers expect image data in the primary HDU and because they can give at a glance an indication of the data quality and an average spectrum.

\subsection{RDR Data Product Details}

The source of the data contained in the Juno-UVS Pipeline RDR data products are the JunoUVS EDR data products, as described above. The next step (nicknamed "Mike") in the JunoUVS data processing pipeline adds pointing and calibration information to the EDR data, but maintains the FITS structure. The resulting RDR data product contains the following extensions:

Calibrated Spectral Image: This is a reconstructed histogram generated from the pixel list data in the EDR data product but with instrumental calibrations applied. This summary image is used as a "quick-look" check on data quality. [Extension $0=$ primary FITS header and data unit (HDU)]

Acquisition List: This dataset contains a list of the generated frame acquisitions as determined from the housekeeping data file. The list includes, for each frame, the instrument frame sequence number, start and stop times, mode type, aperture door and other instrument state information. These data are simply copied as is from the EDR data product. The frame acquisition times and instrument state data contained in this list are used to cross-reference with the pixel list mode data in Extension 2 for purposes of selecting data and checking timing consistency. [Extension 1]

Calibrated Pixel List Mode Data: This dataset contains a calibrated version of the complete pixel list science dataset from the EDR data product, plus propagated estimated errors introduced by the separate calibration steps, plus ancillary spatial location and pointing information that is needed on a per-photon-event basis. These are the primary science data for use in making maps and other pixel list derived science products. [Extension 2]

Ancillary Data: This dataset contains ancillary spatial location and pointing information that varies smoothly and slowly over the Juno orbit. Also included in this extension are other slowly varying instrument-related quantities, such as the detector locations of the fiducial "stim" pixels, a measure of the background dark signal, and data quality flags. Entries in this table are typically separated by $30 \mathrm{~s}$ intervals instead of on a per-photon basis in order to reduce data volume and computation time. [Extension 3]

Calibrated Analog Count Rate: This dataset contains a high-resolution sequence of UV photon count rates as read from the housekeeping data, with corrections for detector deadtime applied. [Extension 4]

Calibrated Digital Count Rate: This dataset contains a high-resolution sequence of UV photon count rates computed from the calibrated pixel list data for each acquisition (nominally the whole orbit), with corrections for detector dead-time applied. [Extension 5]

Pulse Height Distribution (Lyman Alpha): This is the first of three histograms where the bins are arranged as pulse height vs. time. These data are simply copied as is from the corresponding EDR data product. This histogram contains photons whose spectra are recorded on detector columns numbered between 850 and 930 (i.e., Ly $\alpha$ photons). [Extension 6]

Pulse Height Distribution (Stellar): This is the second of three histograms where the bins are arranged as pulse height vs. time. These data are simply copied as is from the corresponding EDR data product. This histogram contains photons whose spectra are recorded on detector columns numbered between 931 and 1770 (i.e., photons at wavelengths longer than Ly $\alpha$ ). [Extension 7]

Pulse Height Distribution (Stim): This is the last of three histograms where the bins are arranged as pulse height vs. time. These data are simply copied as is from the corresponding 
Table 4 Juno-UVS data products

\begin{tabular}{|c|c|c|c|c|c|}
\hline Name & Description & $\begin{array}{l}\text { CODMAC } \\
\text { Level }\end{array}$ & Inputs & Format & $\begin{array}{l}\text { Estimated } \\
\text { Size (MB) }\end{array}$ \\
\hline $\begin{array}{l}\text { Engineering } \\
\text { Level Data }\end{array}$ & $\begin{array}{l}\text { Instrument value science } \\
\text { data ("raw stream" pixel } \\
\text { list) + instrument } \\
\text { housekeeping data }\end{array}$ & 2 & $\begin{array}{l}\text { Instrument data } \\
\text { files }+ \\
\text { engineering } \\
\text { calibration data }\end{array}$ & $\begin{array}{l}\text { FITS (science }+ \\
\text { housekeeping), } \\
\text { binary (memory } \\
\text { dumps) }\end{array}$ & 60 per orbit \\
\hline $\begin{array}{l}\text { Science } \\
\text { Level Data }\end{array}$ & $\begin{array}{l}\text { Calibrated value science } \\
\text { data(tabulated pixel list) } \\
+ \text { ancillary data }\end{array}$ & 3 & $\begin{array}{l}\text { Engineering } \\
\text { Level data+ } \\
\text { science } \\
\text { calibration data }\end{array}$ & FITS & 1200 per orbit \\
\hline $\begin{array}{l}\text { Brightness } \\
\text { Images }\end{array}$ & $\begin{array}{l}\mathrm{N} \& \mathrm{~S} \text { images of UV } \\
\text { brightness at selected } \\
\text { wavelengths \& epochs }+ \\
\text { statistical errors, data } \\
\text { quality }\end{array}$ & 5 & $\begin{array}{l}\text { Science Level } \\
\text { data }\end{array}$ & FITS & 150 per orbit \\
\hline $\begin{array}{l}\text { Brightness } \\
\text { Maps }\end{array}$ & $\begin{array}{l}\text { N \& S polar maps of UV } \\
\text { brightness at selected } \\
\text { wavelengths \& epochs }+ \\
\text { statistical errors, data } \\
\text { quality }\end{array}$ & 5 & $\begin{array}{l}\text { Science Level } \\
\text { data }\end{array}$ & FITS & 300 per orbit \\
\hline $\begin{array}{l}\text { Color Ratio } \\
\text { Maps }\end{array}$ & $\begin{array}{l}\text { N \& S polar maps of UV } \\
\text { color ratio + statistical } \\
\text { and systematic errors and } \\
\text { data quality }\end{array}$ & 5 & $\begin{array}{l}\text { Science Level } \\
\text { data }\end{array}$ & FITS & 50 per orbit \\
\hline $\begin{array}{l}\text { Precipitating } \\
\text { Electron } \\
\text { Energy \& } \\
\text { Flux Maps }\end{array}$ & $\begin{array}{l}\text { N \& S polar maps of } \\
\text { estimated mean energy } \\
\text { and flux of auroral } \\
\text { electrons + statistical and } \\
\text { systematic errors and data } \\
\text { quality }\end{array}$ & 5 & $\begin{array}{l}\text { Science Level } \\
\text { data }+ \text { models }\end{array}$ & FITS & 50 per orbit \\
\hline
\end{tabular}

EDR data product. This histogram contains "photons" whose "spectra" are recorded on detector columns numbered between 0-149 and 1950-2047 (i.e., the stim pulse regions). [Extension 8]

Housekeeping Data: This dataset contains the complete housekeeping dataset, both in raw format and, where applicable, in calibrated engineering units. These data are mostly copied as is from the EDR data product. HK data are included here to assist with joint instrument and data quality trending analyses (foreseen and unforeseen). [Extension 9]

Wavelength Lookup Image: This dataset contains a $2048 \times 256$ image whose floatingpoint pixel values are the wavelengths corresponding to the pixel locations on the detector. This wavelength calibration image is provided to be used with Extension 0 for quick-look checks, but not for scientific analysis. Its file-averaged wavelength solution makes it generally unsuitable to be used with pixel list data. [Extension 10]

\subsection{Higher Level Product Details}

The details of the higher level data products, such as spectral images, brightness maps, color ratio maps, etc. are currently undefined, but are envisioned as simple FITS format files with extensions for image, error, and data quality. Table 4 presents a summary of all the planned data products, and an estimate of their data volume. 


\section{Summary}

The Juno mission has begun its 5-year flight to Jupiter, and Juno-UVS has undergone lowvoltage and high-voltage checkouts (and initial instrument compatibility checks), and is found to be in excellent health. Yearly in-flight calibration observations of hot UV stars are planned for Juno-UVS during the mission to further characterize its in-flight performance. It is expected that the data gathered this instrument will provide an excellent resource for improving our understanding of Jupiter's amazing auroras.

Acknowledgements We wish to thank the entire Juno-UVS engineering, science, and support teams at SwRI (especially David Cronk, Joe Esquivel, Jim Foster and Roy Graham), Sensor Sciences, and the Université de Liège. We also thank the Juno project, especially Phil Morton, Mark Boyles, Maurine Miller (and the rest of the payload team) at JPL, John Ricks at LM for shepherding Juno-UVS through ATLO, and Bill McAlpine at JPL for radiation modeling support. Juno-UVS was funded under contracts NAS703001TONMO710962 and NNM06AA75C to NASA, whose support we gratefully acknowledge. This paper is dedicated to the memory of our dear friend and colleague, Dave Slater.

Open Access This article is distributed under the terms of the Creative Commons Attribution License which permits any use, distribution, and reproduction in any medium, provided the original author(s) and the source are credited.

\section{References}

J.M. Ajello, D.E. Shemansky, W.R. Pryor, A.I.F. Stewart, K.E. Simmons, T. Majeed, J.H. Waite Jr., G.R. Gladstone, D. Grodent, Spectroscopic evidence for high-altitude aurora at Jupiter from Galileo extreme ultraviolet spectrometer and Hopkins ultraviolet telescope observations. Icarus 152, 151-171 (2001)

J.M. Ajello, W. Pryor, L. Esposito, I. Stewart, W. McClintock, J. Gustin, D. Grodent, J.-C. Gérard, J.T. Clarke, The Cassini campaign observations of the Jupiter aurora by the ultraviolet imaging spectrograph and the space telescope imaging spectrograph. Icarus 178, 327-345 (2005)

F. Bagenal, A. Adriani, F. Allegrini, S.J. Bolton, B. Bonfond, E.J. Bunce, J.E.P. Connerney, S.W.H. Cowley, R.W. Ebert, G.R. Gladstone, C.J. Hansen, W.S. Kurth, S.M. Levin, B.H. Mauk, D.J. McComas, C.P. Paranicas, D. Santos-Costa, R.M. Thorne, P. Valek, J.H. Waite, P. Zarka, Space Sci. Rev. (2014, this issue). doi:10.1007/s11214-014-0036-8

A. Bhardwaj, G.R. Gladstone, Auroral emissions of the giant planets. Rev. Geophys. 38, 295-353 (2000)

B. Bonfond, D. Grodent, J.-C. Gérard, A. Radioti, V. Dols, P.A. Delamere, J.T. Clarke, The Io UV footprint: location, inter-spot distances and tail vertical extent. J. Geophys. Res. 114, A07224 (2009)

B. Bonfond, M.F. Vogt, J.-C. Gérard, D. Grodent, A. Radioti, V. Coumans, Quasi-periodic polar flares at Jupiter: a signature of pulsed dayside reconnections? Geophys. Res. Lett. 38, L02104 (2011)

B. Bonfond, D. Grodent, J.-C. Gérard, T. Stallard, J.T. Clarke, M. Yoneda, A. Radioti, J. Gustin, Auroral evidence of Io's control over the magnetosphere of Jupiter. Geophys. Res. Lett. 39, L01105 (2012)

A.L. Broadfoot, M.J.S. Belton, P.Z. Takacs, B.R. Sandel, D.E. Shemansky, J.B. Holberg, J.M. Ajello, S.K. Atreya, T.M. Donahue, H.W. Moos, J.L. Bertaux, J.E. Blamont, D.F. Strobel, J.C. McConnell, A. Dalgarno, R. Goody, M.B. McElroy, Extreme ultraviolet observations from Voyager 1: encounter with Jupiter. Science 204, 979-982 (1979)

J.T. Clarke, G. Ballester, J. Trauger, J. Ajello, W. Pryor, K. Tobiska, J.E.P. Connerney, G.R. Gladstone, J.H. Waite Jr., L. Ben Jaffel, J.-C. Gérard, Hubble space telescope imaging of Jupiter's UV aurora during the Galileo orbiter mission. J. Geophys. Res. 103, 20,217-20,236 (1998)

J.T. Clarke, J.M. Ajello, G. Ballester, L. Ben Jaffel, J.E.P. Connerney, J.-C. Gérard, G.R. Gladstone, D. Grodent, W. Pryor, J. Trauger, J.H. Waite Jr., Ultraviolet emissions from the magnetic footprints of Io, Ganymede, and Europa on Jupiter. Nature 415, 997-1000 (2002)

J.T. Clarke, D. Grodent, S.W.H. Cowley, E.J. Bunce, P. Zarka, J.E.P. Connerney, T. Satoh, Jupiter's aurora, in Jupiter: The Planet, Satellites and Magnetosphere, ed. by F. Bagenal, T. Dowling, W. McKinnon (Cambridge University Press, Cambridge, 2004)

J.T. Clarke, J. Nichols, J.-C. Gérard, D. Grodent, K.C. Hansen, W. Kurth, G.R. Gladstone, J. Duval, S. Wannawichian, E. Bunce, S.W.H. Cowley, F. Crary, M. Dougherty, L. Lamy, D. Mitchell, W. Pryor, K. Retherford, T. Stallard, B. Zieger, P. Zarka, B. Cecconi, Response of Jupiter's and Saturn's auroral activity to the solar wind. J. Geophys. Res. 114, A05210 (2009) 
V. Dols, J.-C. Gérard, J.T. Clarke, J. Gustin, D. Grodent, Diagnostics of the Jovian aurora deduced from ultraviolet spectroscopy: model and HST/GHRS observations. Icarus 147, 251-266 (2000)

J.-C. Gérard, J. Gustin, D. Grodent, J.T. Clarke, A. Grard, Spectral observations of transient features in the FUV Jovian polar aurora. J. Geophys. Res. 108, 1319 (2003)

G.R. Gladstone, Y.L. Yung, An analysis of the reflection spectrum of Jupiter from $1500 \AA$ to $1740 \AA$. Astrophys. J. 266, 415-424 (1983)

G.R. Gladstone, M. Allen, Y.L. Yung, Hydrocarbon photochemistry in the upper atmosphere of Jupiter. Icarus 119, 1-52 (1996)

G.R. Gladstone, S.A. Stern, D.C. Slater, M. Versteeg, M.W. Davis, K.D. Retherford, L.A. Young, A.J. Steffl, H. Throop, H.A. Weaver, A.F. Cheng, G.S. Orton, J.T. Clarke, Jupiter's night side airglow and aurora as seen from New Horizons. Science 317, 229-231 (2007)

G.R. Gladstone, S.A. Stern, K.D. Retherford, R.K. Black, D.C. Slater, M.W. Davis, M.H. Versteeg, K.B. Persson, J.Wm. Parker, D.E. Kaufmann, A.F. Egan, T.K. Greathouse, P.D. Feldman, D. Hurley, W.R. Pryor, A.R. Hendrix, LAMP: the Lyman alpha mapping project on NASA's Lunar reconnaissance orbiter mission. Space Sci. Rev. 150, 161-181 (2010)

D. Grodent, G.R. Gladstone, J.-C. Gérard, V. Dols, J.H. Waite Jr., Simulation of the morphology of the Jovian UV north aurora observed with the Hubble space telescope. Icarus 128, 306-321 (1997)

D. Grodent, J.T. Clarke, J. Kim, J.H. Waite, S.W.H. Cowley, Jupiter's main auroral oval observed with HSTSTIS. J. Geophys. Res. 108, 1389 (2003a)

D. Grodent, J.T. Clarke, J.H. Waite, S.W.H. Cowley, J.-C. Gérard, J. Kim, Jupiter's polar auroral emissions. J. Geophys. Res. 108, 1366 (2003b)

D. Grodent, J.-C. Gérard, J.T. Clarke, G.R. Gladstone, J.H. Waite, A possible auroral signature of a magnetotail reconnection process on Jupiter. J. Geophys. Res. 109, A05201 (2004)

D. Grodent, B. Bonfond, J.-C. Gérard, A. Radioti, J. Gustin, J.T. Clarke, J. Nichols, J.E.P. Connerney, Auroral evidence of a localized magnetic anomaly in Jupiter's northern hemisphere. J. Geophys. Res. 113, A09201 (2008a)

D. Grodent, J.-C. Gérard, A. Radioti, B. Bonfond, A. Saglam, Jupiter's changing auroral location. J. Geophys. Res. 113, A01206 (2008b)

D. Grodent, B. Bonfond, A. Radioti, J.-C. Gérard, X. Jia, J.D. Nichols, J.T. Clarke, Auroral footprint of Ganymede. J. Geophys. Res. 114, A07212 (2009)

J. Gustin, P.D. Feldman, J.-C. Gérard, D. Grodent, A. Vidal-Madjar, L.Ben Jaffel, J.-M. Desert, H.W. Moos, D.J. Sahnow, H.A. Weaver, B.C. Wolven, J.M. Ajello, J.H. Waite, E. Roueff, H. Abgrall, Jovian auroral spectroscopy with FUSE: analysis of self-absorption and implications for electron precipitation. Icarus 171, 336-355 (2004)

J. Gustin, S.W.H. Cowley, J.-C. Gérard, G.R. Gladstone, D. Grodent, J.T. Clarke, Characteristics of Jovian morning bright FUV aurora from Hubble space telescope/space telescope imaging spectrograph imaging and spectral observations. J. Geophys. Res. 111, A09220 (2006)

A.P. Ingersoll et al., Space Sci. Rev. (2014, this issue)

P. Jelinsky, S. Jelinsky, Low reflectance EUV materials: a comparative study. Appl. Opt. 26(4), 613-615 (1987)

T.A. Livengood, H.W. Moos, G.E. Ballester, R.M. Prangé, Jovian ultraviolet auroral activity, 1981-1991. Icarus 97, 26-45 (1992)

B.H. Mauk, J.T. Clarke, D. Grodent, J.H. Waite Jr., C.P. Paranicas, D.J. Williams, Transient aurora on Jupiter from injections of magnetospheric electrons. Nature 415, 1003-1005 (2002)

H. Melin, S. Miller, T. Stallard, C. Smith, D. Grodent, Estimated energy balance in the Jovian upper atmosphere during an auroral heating event. Icarus 181, 256-265 (2006)

K.A. Moldosanov, M.A. Samsonov, L.S. Kim, R. Henneck, O.H.W. Siegmund, J. Warren, S. Cully, D. Marsh, Highly absorptive coating for the vacuum ultraviolet range. Appl. Opt. 37(1), 93-97 (1998)

J.I. Moses, T. Fouchet, B. Bézard, G.R. Gladstone, E. Lellouch, H. Feuchtgruber, Photochemistry and diffusion in Jupiter's stratosphere: constraints from ISO observations and comparisons with other giant planets. J. Geophys. Res. 110, E08001 (2005)

J.D. Nichols, E.J. Bunce, J.T. Clarke, S.W.H. Cowley, J.-C. Gérard, D. Grodent, W.R. Pryor, Response of Jupiter's UV auroras to interplanetary conditions as observed by the Hubble space telescope during the Cassini flyby campaign. J. Geophys. Res. 112, A02203 (2007)

J.D. Nichols, J.T. Clarke, J.-C. Gérard, D. Grodent, Observations of Jovian polar auroral filaments. Geophys. Res. Lett. 36, L08101 (2009a)

J.D. Nichols, J.T. Clarke, J.-C. Gérard, D. Grodent, K.C. Hansen, Variation of different components of Jupiter's auroral emission. J. Geophys. Res. 114, A06210 (2009b)

L. Pallier, R. Prangé, More about the structure of the high latitude Jovian aurorae. Planet. Space Sci. 49, 1159-1173 (2001) 
J.J. Perry, Y.H. Kim, J.L. Fox, H.S. Porter, Chemistry of the Jovian auroral ionosphere. J. Geophys. Res. 104, $16,541-16,566$ (1999)

R. Prangé, D. Rego, D. Southwood, P. Zarka, S. Miller, W.-H. Ip, Rapid energy dissipation and variability of the Io-Jupiter electrodynamic circuit. Nature 379, 323-325 (1996)

W.R. Pryor, A.I.F. Stewart, K.E. Simmons, J.M. Ajello, W.K. Tobiska, J.T. Clarke, G.R. Gladstone, Detection of rapidly varying $\mathrm{H}_{2}$ emissions in Jupiter's aurora from the Galileo orbiter. Icarus 151, 314-317 (2001)

W.R. Pryor, A.I.F. Stewart, L.W. Esposito, W.E. McClintock, J.E. Colwell, A.J. Jouchoux, A.J. Steffl, D.E. Shemansky, J.M. Ajello, R.A. West, C.J. Hansen, B.T. Tsurutani, W.S. Kurth, G.B. Hospodarsky, D.A. Gurnett, K.C. Hansen, J.H. Waite Jr., F.J. Crary, D.T. Young, N. Krupp, J.T. Clarke, D. Grodent, M.K. Dougherty, Cassini UVIS observations of Jupiter's auroral variability. Icarus 178, 312-326 (2005)

W.R. Pryor, P. Gangopadhyay, B. Sandel, T. Forrester, E. Quemerais, E. Moebius, L. Esposito, A.I.F. Stewart, B. McClintock, A. Jouchoux, J. Colwell, V. Izmodenov, Y. Malena, W.K. Tobiska, D.E. Shemansky, J.M. Ajello, C. Hansen, M. Bzowski, P. Frisch, Radiation transport of heliospheric Lyman-alpha from combined Cassini and Voyager data sets. Astron. Astrophys. 491, 21-28 (2008)

A. Radioti, D. Grodent, J.-C. Gérard, B. Bonfond, J.T. Clarke, Auroral polar dawn spots: signatures of internally driven reconnection processes at Jupiter's magnetotail. Geophys. Res. Lett. 35, L03104 (2008)

A. Radioti, A.T. Tomás, D. Grodent, J.-C. Gérard, J. Gustin, B. Bonford, N. Krupp, J. Woch, J.D. Menietti, Equatorward diffuse auroral emissions at Jupiter: simultaneous HST and Galileo observations. Geophys. Res. Lett. 36, L07101 (2009)

A. Radioti, D. Grodent, J.-C. Gérard, B. Bonfond, Auroral signatures of flow bursts released during magnetotail reconnection at Jupiter. J. Geophys. Res. 115, A07214 (2010)

A. Radioti, D. Grodent, J.-C. Gérard, M.F. Vogt, M. Lystrup, B. Bonfond, Nightside reconnection at Jupiter: auroral and magnetic field observations from 26 July 1998. J. Geophys. Res. 116, A03221 (2011)

A. Seiff, D.B. Kirk, T.C.D. Knight, R.E. Young, J.D. Mihalov, L.A. Young, F.S. Milos, G. Schubert, R.C. Blanchard, D. Atkinson, Thermal structure of Jupiter's atmosphere near the edge of a $5-\mu \mathrm{m}$ hot spot in the north equatorial belt. J. Geophys. Res. 103, 22857-22889 (1998)

O.H.W. Siegmund, Microchannel plate imaging detector technologies for UV instruments, in From X-Rays to $X$-Band-Space Astrophysics Detectors and Detector Technologies (Space Telescope Science Institute, Baltimore, 2000)

O.H.W. Siegmund, P. Jelinsky, S. Jelinsky, J. Stock, J. Hull, D. Doliber, J. Zaninovich, A.S. Tremsin, K. Kromer, High resolution cross delay line detectors for the GALEX mission. Proc. SPIE 3765, 429-440 (1999)

D.C. Slater, S.A. Stern, T. Booker, J. Scherrer, M.F. A'Hearn, J.L. Bertaux, P.D. Feldman, M.C. Festou, O.H.W. Siegmund, Radiometric and calibration performance results for the Rosetta UV imaging spectrometer Alice. Proc. SPIE 4498, 239-247 (2001)

D.C. Slater, M.W. Davis, C.B. Olkin, S.A. Stern, J. Scherrer, Radiometric performance results of the New Horizons' Alice UV imaging spectrograph. Proc. SPIE 5906, 368-379 (2005)

S.A. Stern, D.C. Slater, W. Gibson, J. Scherrer, M. A'Hearn, J.-L. Bertaux, P.D. Feldman, M.C. Festou Alice, An ultraviolet imaging spectrometer for the Rosetta orbiter. Adv. Space Res. 21, 1517-1525 (1998)

S.A. Stern, D.C. Slater, J.R. Scherrer, J.M. Stone, G.J. Dirks, M.H. Versteeg, M.W. Davis, G.R. Gladstone, J.Wm. Parker, L.A. Young, O.H.W. Siegmund Alice, The ultraviolet imaging spectrograph aboard the New Horizons Pluto-Kuiper Belt mission. Space Sci. Rev. 140, 155-187 (2008)

L.M. Trafton, V. Dols, J.-C. Gérard, J.H. Waite Jr., G.R. Gladstone, G. Munhoven, HST spectra of the Jovian ultraviolet aurora: search for heavy ion precipitation. Astrophys. J. 507, 955-967 (1998)

M.F. Vogt, M.G. Kivelson, K.K. Khurana, R.J. Walker, B. Bonfond, D. Grodent, A. Radioti, Improved mapping of Jupiter's auroral features to magnetospheric sources. J. Geophys. Res. 116, A03220 (2011)

J.H. Waite Jr., G.R. Gladstone, W.S. Lewis, R. Goldstein, D.J. McComas, P. Riley, R.J. Walker, P. Robertson, S. Desai, J.T. Clarke, D.T. Young, An auroral flare at Jupiter. Nature 410, 787-789 (2001)

S. Wannawichian, J.T. Clarke, J.D. Nichols, Ten years of Hubble space telescope observations of the variation of the Jovian satellites' auroral footprint brightness. J. Geophys. Res. 115, A02206 (2010)

A.-S. Wong, A.Y.T. Lee, Y.L. Yung, J.M. Ajello, Jupiter: aerosol chemistry in the polar atmosphere. Astrophys. J. 534, L215-L217 (2000)

A.-S. Wong, Y.L. Yung, A.J. Friedson, Benzene and haze formation in the polar atmosphere of Jupiter. Geophys. Res. Lett. (2003). doi:10.1029/2002GL016661

Y.L. Yung, G.R. Gladstone, K.M. Chang, J.M. Ajello, S.K. Srivastava, $\mathrm{H}_{2}$ fluorescence spectrum from 1200 to $1700 \AA$ by electron impact: laboratory study and application to Jovian aurora. Astrophys. J. 254, L65-L69 (1982) 